\title{
Wave-Corpuscle Mechanics for Electric Charges
}

\author{
Anatoli Babin · Alexander Figotin
}

Received: 14 December 2008 / Accepted: 10 November 2009 / Published online: 19 November 2009

(C) The Author(s) 2009. This article is published with open access at Springerlink.com

\begin{abstract}
It is well known that the concept of a point charge interacting with the electromagnetic (EM) field has a problem. To address that problem we introduce the concept of wave-corpuscle to describe spinless elementary charges interacting with the classical EM field. Every charge interacts only with the EM field and is described by a complex valued wave function over the 4-dimensional space time continuum. A system of many charges interacting with the EM field is defined by a local, gauge and Lorentz invariant Lagrangian with a key ingredient - a nonlinear self-interaction term providing for a cohesive force assigned to every charge. An ideal wave-corpuscle is an exact solution to the Euler-Lagrange equations describing both free and accelerated motions. It carries explicitly features of a point charge and the de Broglie wave. Our analysis shows that a system of well separated charges moving with nonrelativistic velocities are represented accurately as wave-corpuscles governed by the Newton equations of motion for point charges interacting with the Lorentz forces. In this regime the nonlinearities are "stealthy" and don't show explicitly anywhere, but they provide for the binding forces that keep localized every individual charge. The theory can also be applied to closely interacting charges as in hydrogen atom where it produces discrete energy spectrum.
\end{abstract}

Keywords Wave mechanics · Corpuscle · Extended charge - Lorentz-Abraham model · Nonlinearity $\cdot$ Maxwell equations

\section{Introduction}

We all know from textbooks that if there is a point charge $q$ of a mass $m$ in an external electromagnetic (EM) field its non-relativistic dynamics is governed by the equation

$$
\frac{\mathrm{d}}{\mathrm{d} t}[m \mathbf{v}(t)]=q\left[\mathbf{E}(t, \mathbf{r}(t))+\frac{1}{\mathrm{c}} \mathbf{v}(t) \times \mathbf{B}(t, \mathbf{r}(t))\right]
$$

A. Babin · A. Figotin (凶)

University of California at Irvine, Irvine, USA

e-mail: afigotin@math.uci.edu
A. Babin
e-mail: ababine@math.uci.edu 
where $\mathbf{r}$ and $\mathbf{v}=\dot{\mathbf{r}}=\frac{\mathrm{d} \mathbf{r}}{\mathrm{d} t}$ are respectively the charge's time-dependent position and velocity, $\mathbf{E}(t, \mathbf{r})$ and $\mathbf{B}(t, \mathbf{r})$ are the electric field and the magnetic induction, $\mathrm{c}$ is speed of light and the right-hand side of (1) is the Lorentz force. We also know that if the charge's time-dependent position and velocity are $\mathbf{r}$ and $\mathbf{v}$ then there is associated with them an EM field described by the equations

$$
\begin{aligned}
\frac{1}{\mathrm{c}} \frac{\partial \boldsymbol{B}}{\partial t}+\nabla \times \boldsymbol{E} & =\mathbf{0}, \quad \nabla \cdot \boldsymbol{B}=0, \\
\frac{1}{\mathrm{c}} \frac{\partial \boldsymbol{E}}{\partial t}-\nabla \times \boldsymbol{B} & =-\frac{4 \pi}{\mathrm{c}} q \delta(\mathbf{x}-\mathbf{r}(t)) \mathbf{v}(t), \\
\nabla \cdot \boldsymbol{E} & =4 \pi q \delta(\mathbf{x}-\mathbf{r}(t)),
\end{aligned}
$$

where $\delta$ is the Dirac delta-function, $\mathbf{v}(t)=\dot{\mathbf{r}}(t)$. But if naturally we would like to consider (1) and (2)-(3) as a closed system "charge-EM field" there is a problem. The origin of the problems is in the divergence of the EM field exactly at the position of the point charge, as, for instance, for the electrostatic field $\mathbf{E}$ with the Coulomb's potential $\frac{q}{|\mathbf{x}-\mathbf{r}|}$ with a singularity at $\mathbf{x}=\mathbf{r}$. If in (1) $m$ replaced with $\gamma m$, where $\gamma=1 / \sqrt{1-\mathbf{v}^{2}(t) / \mathrm{c}^{2}}$ is the Lorentz factor, the system constituted by the modified equation and (2)-(3) becomes Lorentz invariant. In fact, the modified system has a Lagrangian that yields it via the variational principle [7, (4.21)], [41, (2.36)], but the problem still persists. Some studies indicate that, [49], "a fully consistent classical equation of motion for a point charge, unlike that of an extended charge, does not exist". If one wants to stay within the classical theory of electromagnetism, a possible remedy is the introduction of an extended charge which, though very small, is not a point. There are two most well known models for such an extended charge: the semi-relativistic Abraham rigid charge model (a rigid sphere with spherically symmetric charge distribution), [41, Sects. 2.4, 4.1, 10.2, 13], [37, Sects. 2.2], and the Lorentz relativistically covariant model which was studied and advanced in [1], [23, Sect. 16], [31], [34], [37, Sects. 2, 6], [40], [41, Sects. 2.5, 4.2, 10.1], [48]. Importantly for what we do here, Poincaré suggested in 1905-1906, [35, 36] (see also [23, Sects. 16.4-16.6], [37, Sects. 2.3, 6.1-6.3], [33, Sect. 63], [40], [48, Sect. 4.2] and references there in), to add to the Lorentz-Abraham model nonelectromagnetic cohesive forces which balance the charge internal repulsive electromagnetic forces and remarkably restore also the covariance of the entire model. W. Pauli argues very convincingly based on the relativity principle in [33, Sect. 63] the necessity to introduce for the electron an energy of non-electromagnetic origin.

An alternative approach to deal with the above-mentioned divergences goes back to G. Mie who proposed to modify the Maxwell equations making them nonlinear, [33, Sect. 64], [47, Sect. 26] and a particular example of the Mie approach is the Born-Infeld theory, [12]. Recently M. Kiessling showed that, [25], "a relativistic Hamilton-Jacobi type law of point charge motion can be consistently coupled with the nonlinear Maxwell-BornInfeld field equations to obtain a well-defined relativistic classical electrodynamics with point charges".

A substantially different approach to elementary charges was pursued by E. Schrödinger who tried to develop a concept of wavepacket as a model for spatially localized charge. The Schrödinger wave theory, [39], was inspired by de Broglie ideas, [13], [7, Sect. II.1]. The theory was very successful in describing quantum phenomena in the hydrogen atom, but it had great difficulties in treating the elementary charge as the material wave as it moves and interacts with other elementary charges. M. Born commented on this, [11, Chap. IV.7]: "To begin with, Schrödinger attempted to interpret corpuscles and particularly electrons, as wave packets. Although his formulae are entirely correct, his interpretation cannot be maintained, 
since on the one hand, as we have already explained above, the wave packets must in course of time become dissipated, and on the other hand the description of the interaction of two electrons as a collision of two wave packets in ordinary three-dimensional space lands us in grave difficulties."

The wave-corpuscule mechanics (WCM) is a Lagrangian mechanics that includes as an important element a concept of wave-corpuscle. The wave-corpuscle is understood as a spatially localized solution to a nonlinear Schroedinger or Klein-Gordon equation, if we neglect spin. Our approach to a spatially distributed but localized elementary charge has some features in common with the above discussed concepts of extended charge, but it differs from any of them substantially. In particular, our approach provides for an electromagnetic theory in which (i) a "bare" elementary charge and the EM field described by the Maxwell equations form an inseparable entity; (ii) every elementary "bare" charge interacts directly only with the EM field; (iii) the EM field is a single entity providing for the interaction between "bare" elementary charges insuring the maximum speed of interaction not to ever exceed the speed of light. To emphasize the inseparability of the "bare" elementary charge from the EM field we refer to their entity as to dressed charge.

The best way to describe our concept of a spatially distributed but localized dressed charge in one word is by the name wave-corpuscle, since it is a stable localized excitation of a dispersive medium propagating in the three-dimensional space. An instructive example of a wave-corpuscle is furnished by our nonrelativistic charge model. In that model, in the simplest case, an ideal wave-corpuscle is described by a complex-valued wave function $\psi$ of the form

$$
\psi=\psi(t, \mathbf{x})=\exp \left\{\frac{\mathrm{i}}{\hbar}\left[\mathbf{p}(t) \cdot(\mathbf{x}-\mathbf{r}(t))+s_{\mathrm{p}}(t)\right]\right\} \stackrel{\circ}{\psi}(|\mathbf{x}-\mathbf{r}(t)|),
$$

where $\dot{\psi}(s), s \geq 0$, is a non-negative, monotonically decaying function which vanishes at infinity at a sufficiently fast rate, $s_{\mathrm{p}}(t)$ is a phase shift and $\mathbf{E}_{\mathrm{ex}}(t)$ is an external homogeneous electric field. Importantly, for the above wave function $\psi$ to be an exact solution of corresponding field equations, the parameters $\mathbf{r}(t)$ and $\mathbf{p}(t)$ satisfy the Newton's equations which in this simplest case have the form

$$
m \frac{\mathrm{d}^{2} \mathbf{r}(t)}{\mathrm{d} t^{2}}=q \mathbf{E}_{\mathrm{ex}}, \quad \mathbf{p}(t)=m \frac{\mathrm{d} \mathbf{r}(t)}{\mathrm{d} t},
$$

where $m$ and $q$ are respectively its mass and the charge. We would like to emphasize that the Newton's equations are not postulated as in (1) but rather are derived from the field equations. In contrast to Abraham and Lorentz models as well as quantum Abraham models with de Broglie-Bohm laws of quantum motion introduced by M. Kiessling in [26] we do not define a charge as an object with prescribed geometry, the dynamics and shape of a wave corpuscle is governed by a nonlinear Klein-Gordon or a nonlinear Schrödinger equation in relativistic and nonrelativistic cases respectively and a wave-corpuscle is defined as a special type of solutions to these equations.

The ideal wave-corpuscle wave function $\psi(t, \mathbf{x})$ defined by (4), (5) together with the corresponding EM field forms an exact solution to the relevant Euler-Lagrange field equations describing an accelerating dressed charge. The point charge momentum $\mathbf{p}(t)$ turns out to be exactly equal to the total momentum of the charge as a wave-corpuscle and its electromagnetic field. The wave-corpuscle is a material wave in space, more precisely a field on space-time, and the quantity $q|\psi(t, \mathbf{x})|^{2}$ corresponds to the charge density and the density $|\psi(t, \mathbf{x})|^{2}$ is not given a probabilistic interpretation. The WCM can be characterized in 
one word as "neo-classical" as suggested by Michael Kiessling. We believe that our wavecorpuscle provides an alternative resolution to the wave-particle duality problem.

We develop here both the relativistic and non-relativistic versions of the theory for one and many charges. The field equations of the relativistic theory include, in particular, the classical Maxwell equations

$$
\partial_{\mu} F^{\mu v}=\frac{4 \pi}{\mathrm{c}} J^{v}, \quad F^{\mu v}=\partial^{\mu} A^{v}-\partial^{v} A^{\mu},
$$

where $A^{v}=(\varphi, \mathbf{A})$ is the 4-potential, $F^{\mu \nu}$ is the antisymmetric tensor of EM field, and $J^{\nu}=(\mathrm{c} \varrho, \mathbf{J})$ is the 4-current composed of all conserved 4-currents of individual charges. The dynamics of charges is determined by nonlinear Klein-Gordon field equations, instead of Newton's equations with Lorentz forces. Though the WCM covers all spatial scales the primary focus of this paper is on the case when charges are well separated, and we only briefly discuss the hydrogen atom model as well as a comparison of the WCM with quantum mechanics.

To keep the size of the paper within reasonable limits we had to omit many details related to symmetries, gauge invariance, conservation laws as well as derivations of expressions for densities of the energy-momentum tensor, angular momentum tensor, currents, internal forces all of which a curious reader can find in our manuscript [6].

\section{Nonrelativistic Theory for the Charge in an External EM Field}

The nonrelativistic case, i.e. the case when a charge moves with a velocity much smaller than the velocity of light, is important for our studies for at least two reasons. First of all, we need it to relate the wave-corpuscle mechanics to the Newtonian mechanics for point charges in EM fields. Second of all, in the nonrelativistic case we can carry out rather detailed analytical studies of many physical quantities in a closed form. With that in mind, we would like to treat the nonrelativistic case not just as an approximation to the relativistic theory but rather as a case on its own, and we do it by constructing a certain nonrelativistic Lagrangian $\hat{L}_{0}$ intimately related to the relativistic Lagrangian defined in (153). Some details of the relation between the relativistic and nonrelativistic Lagrangians are considered in Sect. 3.3.

\subsection{Lagrangian and Field Equations}

Our nonrelativistic Lagrangian for a single charge in an external EM field with potentials $\varphi_{\mathrm{ex}}, \mathbf{A}_{\mathrm{ex}}$ has the form

$$
\begin{aligned}
\hat{L}_{0}\left(\psi, \psi^{*}, \varphi\right) & =\frac{\chi}{2} \mathrm{i}\left[\psi^{*} \tilde{\partial}_{t} \psi-\psi \tilde{\partial}_{t}^{*} \psi^{*}\right]-\frac{\chi^{2}}{2 m}\left\{\tilde{\nabla} \psi \tilde{\nabla}^{*} \psi^{*}+G\left(\psi^{*} \psi\right)\right\}+\frac{|\nabla \varphi|^{2}}{8 \pi}, \\
\tilde{\partial}_{t} & =\partial_{t}+\frac{\mathrm{i} q\left(\varphi+\varphi_{\mathrm{ex}}\right)}{\chi}, \quad \tilde{\nabla}=\nabla-\frac{\mathrm{i} q \mathbf{A}_{\mathrm{ex}}}{\chi c},
\end{aligned}
$$

where $\psi^{*}$ is complex conjugate to $\psi, m>0$ is the charge mass, $\chi>0$ is a constant similar to the Planck constant $\hbar=\frac{h}{2 \pi}$, and $q$ is the charge. The Lagrangian expression indicates that the charge is coupled to the EM field through the covariant derivatives, and that type of coupling is known as minimal. Evidently in this Lagrangian the EM field of the charge is represented only by its scalar potential $\varphi$ and the corresponding electric field $\mathbf{E}=-\nabla \varphi$, 
and the charge's magnetic field is assumed to be identically zero. Consequently, any radiation phenomena are excluded in this model. The Lagrangian $\hat{L}_{0}$ can be viewed as a field version of the point charges model that neglects all retardation effects in the static limit (zeroth order in $\frac{v}{c}$ ) with the "instantaneous" interaction Lagrangian $-\frac{q_{1} q_{2}}{\left|\mathbf{r}_{1}-\mathbf{r}_{2}\right|}$ between two charges, [23, Sect. 12.6]. More detailed discussions on the relations between relativistic and nonrelativistic Lagrangians are provided in Sect. 3.3.

The field equations for this case take the form of the following nonlinear Schrödinger equation for $\psi$ coupled with the equation for the electric potential $\varphi$ :

$$
\begin{aligned}
\mathrm{i} \chi \partial_{t} \psi & =-\frac{\chi^{2} \nabla^{2} \psi}{2 m}-\frac{\chi q \mathbf{A}_{\mathrm{ex}} \cdot \nabla \psi}{m \mathrm{c} i}+q\left(\varphi+\varphi_{\mathrm{ex}}+\frac{q \mathbf{A}_{\mathrm{ex}}^{2}}{2 m \mathrm{c}^{2}}\right) \psi+G_{a}^{\prime}\left(|\psi|^{2}\right) \psi, \\
-\nabla^{2} \varphi & =4 \pi q|\psi|^{2},
\end{aligned}
$$

where $G^{\prime}(s)=\partial_{s} G$, and we refer to the pair $\{\psi, \varphi\}$ as dressed charge. The integral of $|\psi|^{2}$ over the entire space turns to be a conserved quantity which we set to be 1 , namely

$$
\int_{\mathbb{R}^{3}}|\psi|^{2} \mathrm{~d} \mathbf{x}=1
$$

and we refer to the above equality as the charge normalization condition.

Let us consider now the case of a resting charge without external EM field, namely with $\mathbf{A}_{\mathrm{ex}}=0, \varphi_{\mathrm{ex}}=0$ in (9). We describe the resting charge by time independent real-valued radial functions $\stackrel{\psi}{\psi}=\stackrel{\wp}{\psi}(|\mathbf{x}|)$ and $\stackrel{\circ}{\varphi}=\stackrel{\circ}{\varphi}(|\mathbf{x}|)$ that solve (9)-(10) which turn into the following rest charge equations:

$$
\begin{aligned}
& -\nabla^{2} \stackrel{\circ}{\varphi}=4 \pi q|\dot{\psi}|^{2}, \\
& -\nabla^{2} \stackrel{\circ}{\psi}+\frac{2 m}{\chi^{2}} q \stackrel{\circ}{\varphi} \dot{\psi}+G^{\prime}\left(|\stackrel{\psi}{\psi}|^{2}\right) \stackrel{\circ}{\psi}=0 .
\end{aligned}
$$

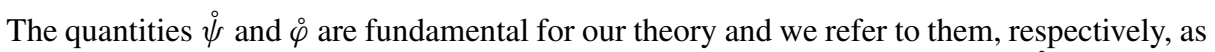
form factor and form factor potential. In view of (12) the charge form factor $\stackrel{\psi}{\psi}$ determines

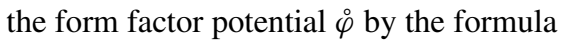

$$
\stackrel{\circ}{\varphi}(|\mathbf{x}|)=\stackrel{\circ}{\varphi}_{\dot{\psi}}(|\mathbf{x}|)=q \int_{\mathbb{R}^{3}} \frac{\stackrel{\leftrightarrow}{ }^{2}(|\mathbf{y}|)}{|\mathbf{y}-\mathbf{x}|} \mathrm{d} \mathbf{y},
$$

and if we plug in the above expression into (13), we get the following nonlinear equation

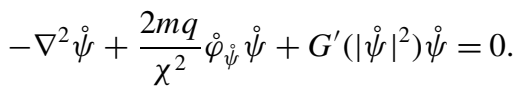

Equation (15) signifies a complete balance of the three forces acting upon the resting charge: (i) internal elastic deformation force associated with the term $-\Delta \psi$; (ii) the charge's electromagnetic self-interaction force associated with the term $\frac{2 m q}{\chi^{2}} \stackrel{\circ}{\varphi}_{\psi} \stackrel{\circ}{\psi}$; (iii) internal nonlinear self-interaction of the charge associated with the term $G^{\prime}\left(|\stackrel{\circ}{\psi}|^{2}\right) \dot{\psi}$. We refer to (15), which establishes an explicit relation between the form factor $\psi$ and the self-interaction nonlinearity $G$, as the charge equilibrium equation. Hence, being given the form factor $\dot{\psi}$, we can find from the equilibrium equation (15) the self-interaction nonlinearity $G$ which exactly produces this factor under the assumption that $\psi(r)$ is a nonnegative, monotonically decaying and sufficiently smooth function of $r \geq 0$. Thus, we pick the form factor $\psi$, and then the 
nonlinear self interaction function $G$ is determined based on the charge equilibrium equation (15). The later is a key feature of our approach: it allows to choose the form factor $\dot{\psi}$ and then to determine matching self-interaction nonlinearity $G$ rather than to deal with solving a nontrivial nonlinear partial differential equation. Note that after the nonlinearity $G$ is determined, it is fixed forever, and while solutions of (9)-(10) may evolve in time, they do not need to coincide with $\{\dot{\psi}, \stackrel{\circ}{\varphi}\}$. It is worth to point out that the nonlinearity $G$ introduced via the charge equilibrium equation (136) differs significantly from nonlinearities considered in similar problems in literature including attempts to introduce nonlinearity in the quantum mechanics, [9, 22, 45]. Important features of our nonlinearity include: (i) the boundedness of $G^{\prime}(s)$ for $s \geq 0$ from below with consequent boundedness from below of the wave energy; (ii) non analytic behavior about $s=0$ that is for vanishing wave amplitudes. Details and examples of the construction of the nonlinear self-interaction function $G$ based on the form factor are provided in Sect. 2.4.

We introduce for the form factor its size representation involving size parameter $a>0$ and normalization constant $\stackrel{\circ}{C}$ :

$$
\begin{aligned}
& \dot{\psi}(s)=\stackrel{\circ}{\psi}_{a}(s)=\frac{\stackrel{\circ}{C}}{a^{3 / 2}} \stackrel{\circ}{\psi}_{1}\left(\frac{s}{a}\right), \\
& \stackrel{\circ}{\varphi}(s)=\stackrel{\circ}{\varphi}_{a}(s)=\frac{q}{a} \stackrel{\circ}{\varphi}_{1}\left(\frac{s}{a}\right) \quad \text { if } s \geq 0,
\end{aligned}
$$

where the function $\AA_{1}(|\mathbf{x}|)$ satisfies the normalization condition,

$$
\int_{\mathbb{R}^{3}} \stackrel{\circ}{1}_{1}^{2}(|\mathbf{x}|) \mathrm{d} \mathbf{x}=1
$$

The corresponding nonlinearity $G^{\prime}\left(|\psi|^{2}\right)$ also depends on the size parameter $a, G^{\prime}\left(|\psi|^{2}\right)=$ $G_{a}^{\prime}\left(|\psi|^{2}\right)$, and the explicit form of the dependence is given below in (52).

Let us associate with the field equations (9)-(10) the following complementary point charge equations of motion

$$
\begin{aligned}
& m \frac{\mathrm{d}^{2} \mathbf{r}}{\mathrm{d}^{2} t}=q \mathbf{E}_{\mathrm{ex}}(t, \mathbf{r})+\frac{q}{\mathrm{c}} \frac{\mathrm{d} \mathbf{r}}{\mathrm{d} t} \times \mathbf{B}_{\mathrm{ex}}(t, \mathbf{r}), \\
& \mathbf{r}(0)=\mathbf{r}_{0}, \quad \frac{\mathrm{d} \mathbf{r}}{\mathrm{d} t}(0)=\dot{\mathbf{r}}_{0},
\end{aligned}
$$

where external fields $\mathbf{E}_{\mathrm{ex}}$ and $\mathbf{B}_{\mathrm{ex}}$ are related to potentials $\varphi_{\mathrm{ex}}$ and $\mathbf{A}_{\mathrm{ex}}$ by standard formulas (131) and $\mathbf{r}_{0}, \dot{\mathbf{r}}_{0}$ are initial data. Obviously, (18) have the form of Newton's law of motion for a point charge with Lorentz force as in (1). We define now the wave-corpuscle $\psi, \varphi$ by the following formula:

$$
\begin{aligned}
\psi(t, \mathbf{x}) & =\mathrm{e}^{\mathrm{i} S / \chi} \hat{\psi}, \quad \text { where } S=m \mathbf{v} \cdot(\mathbf{x}-\mathbf{r})+s_{\mathrm{p}}(t), \\
\hat{\psi} & =\stackrel{\circ}{\psi}(|\mathbf{x}-\mathbf{r}|), \quad \text { where } \varphi=\stackrel{\circ}{\varphi}(|\mathbf{x}-\mathbf{r}|), \mathbf{r}=\mathbf{r}(t) .
\end{aligned}
$$

In the above formula $\stackrel{\leftrightarrow}{\psi}$ and $\stackrel{\circ}{\varphi}$ are, respectively, the form factor and the form factor potential satisfying (12), (13). The functions $\mathbf{v}(t), s_{\mathrm{p}}(t)$ in (19) are determined based on the solution 
$\mathbf{r}(t)$ of (18) by the formulas

$$
\begin{aligned}
\mathbf{v}(t) & =\frac{\mathrm{d} \mathbf{r}}{\mathrm{d} t}(t)+\frac{q}{m \mathrm{c}} \mathbf{A}_{\mathrm{ex}}(t, \mathbf{r}(t)), \\
\frac{\mathrm{d} s_{\mathrm{p}}}{\mathrm{d} t} & =\frac{m \mathbf{v}^{2}(t)}{2}-q \varphi_{\mathrm{ex}}(t, \mathbf{r}(t)) .
\end{aligned}
$$

Notice that the function $\mathbf{v}(t)$ defined by the first equation in (20) if $\mathbf{A}_{\mathrm{ex}} \neq 0$ is not the charge velocity $\dot{\mathbf{r}}(t)$, but it is simply related to the canonical momentum $\stackrel{\mathbf{p}}{\text { by }}$ the formula

$$
\begin{aligned}
\mathbf{v}(t) & =\frac{\stackrel{\circ}{\mathbf{p}}(t)}{m}, \quad \text { where } \stackrel{\circ}{\mathbf{p}}=\mathbf{p}+\frac{q}{\mathrm{c}} \mathbf{A}_{\mathrm{ex}}, \\
\text { and } \quad \mathbf{p} & =m \frac{\mathrm{d} \mathbf{r}}{\mathrm{d} t} \quad \text { is the kinetic momentum. }
\end{aligned}
$$

Below we show that if the function $\mathbf{r}=\mathbf{r}(t)$ satisfies (18) then under certain conditions on the external field formula (19) defines an exact solution to the field equations (9)-(10). We refer to the function $\mathbf{r}(t)$ as wave-corpuscle center or wave-corpuscle position. Since $\hat{\psi}$ is center-symmetric, this definition agrees with more general definition (88). Note that in a simpler case when the external fields $\varphi_{\mathrm{ex}}$ and $\mathbf{A}_{\mathrm{ex}}$ vanish, a simpler solution of (18) is $\mathbf{r}(t)=\mathbf{r}_{0}+\mathbf{v} t$ with constant velocity $\mathbf{v}$. In this case the wave-corpuscle solution (19) of the field equations (9)-(10) can be obtained from the rest solution $\stackrel{\leftrightarrow}{\psi}, \stackrel{\circ}{\text { of }}$ (12)-(13) by a certain Galilean-gauge transformations defined in (103)-(104). Solutions of a similar form are known in the theory of Nonlinear Schrödinger equations, see [42] and references therein.

\subsection{Accelerated Motion of Wave-Corpuscle in an External Electric Field}

In this subsection we consider properties of a wave-corpuscle subjected to a purely electric external EM field, i.e. when $\mathbf{A}_{\mathrm{ex}}=0$. We also present here a derivation of (18) based on the requirement that (19) gives an exact solution to the field equations (9), (10). If the external field is purely electric the field equations (9), (10) take the form

$$
\begin{aligned}
\mathrm{i} \chi \partial_{t} \psi & =-\frac{\chi^{2} \nabla^{2} \psi}{2 m}+q\left(\varphi+\varphi_{\mathrm{ex}}\right) \psi+\frac{\chi^{2}}{2 m} G^{\prime}\left(|\psi|^{2}\right) \psi, \\
\nabla^{2} \varphi & =-4 \pi q|\psi|^{2} .
\end{aligned}
$$

In this case the wave-corpuscle is defined by the formula (19) with the complementary point charge equations (18) taking the form

$$
m \frac{\mathrm{d}^{2} \mathbf{r}(t)}{\mathrm{d} t^{2}}=q \mathbf{E}_{\mathrm{ex}}(t, \mathbf{r}),
$$

where $\mathbf{E}_{\mathrm{ex}}(t, \mathbf{x})=-\nabla \varphi_{\mathrm{ex}}(t, \mathbf{x})$. With the initial data $\mathbf{r}_{0}$ and $\dot{\mathbf{r}}_{0}$ as in (18) formula (20) takes the form

$$
\mathbf{v}=\frac{\mathrm{d} r}{\mathrm{~d} t}, \quad \text { and } \quad s_{\mathrm{p}}=\int_{0}^{t}\left(\frac{m \mathbf{v}^{2}}{2}-q \varphi_{\mathrm{ex}}(t, \mathbf{r}(t))\right) \mathrm{d} t^{\prime} .
$$

In the case when the external electric field is spatially homogeneous we show below that the wave-corpuscle is an exact solution to the field equations (22). In fact, formula (19) 
provides also an exact solution for the EM fields with non-zero, spatially constant magnetic field $\mathbf{B}_{\mathrm{ex}}(t)$, and in the latter case the Lorentz force involves $\mathbf{B}_{\mathrm{ex}}$ as in (18).

Looking at the exact solution (19) to the field equations that describes the accelerating charge we would like to acknowledge the truly remarkable simplicity and transparency of the relations between the two concepts of the charge: charge as a field $\{\psi, \varphi\}$ in (19) and charge as a point described by (23) and (24). Indeed, the wave amplitude $\psi(|\mathbf{x}-\mathbf{r}(t)|)$ in (19) is a soliton-like field moving exactly as the point charge described by its position $\mathbf{r}(t)$. The exponential factor $\mathrm{e}^{\mathrm{i} S / \chi}$ is just a plane wave with the phase $S$ that depends only on the point charge position $\mathbf{r}$ and momentum $\mathbf{p}$ and a time dependent gauge term, and it does not depend on the nonlinear self-interaction. The phase $S$ has a term in which we readily recognize the de Broglie wave-vector $\mathbf{k}(t)$ described exactly in terms of the point charge quantities, namely

$$
\chi \mathbf{k}(t)=\mathbf{p}(t)=m \mathbf{v}(t) .
$$

Notice that the dispersion relation $\omega=\omega(\mathbf{k})$ of the linear kinetic part of the field equations (9) for $\psi$ is

$$
\omega(\mathbf{k})=\frac{\chi \mathbf{k}^{2}}{2 m}, \quad \text { implying that the group velocity } \nabla_{\mathbf{k}} \omega(\mathbf{k})=\frac{\chi \mathbf{k}}{m} .
$$

Combining the expression (26) for the group velocity $\nabla_{\mathbf{k}} \omega(\mathbf{k})$ with the expression (25) for wave vector $\mathbf{k}(t)$ we establish another exact relation, namely

$$
\mathbf{v}(t)=\nabla_{\mathbf{k}} \omega(\mathbf{k}(t)),
$$

signifying the equality between the point charge velocity $\mathbf{v}(t)$ and the group velocity $\nabla_{\mathbf{k}} \omega(\mathbf{k}(t))$ at the de Broglie wave vector $\mathbf{k}(t)$. Similar analysis for the case of non-zero spatially homogeneous magnetic field is given in [6].

To summarize the above analysis we may state that even when the charge accelerates it perfectly combines the properties of a wave and a corpuscle, justifying the name wavecorpuscle mechanics. Its wave nature manifests itself, in particular, in the de Broglie exponential factor and the equality (27), indicating the wave origin the charge motion. The corpuscle properties are manifested in the factor $\psi(|\mathbf{x}-\mathbf{r}(t)|)$ and in the soliton like propagation with $\mathbf{r}(t)$ satisfying the classical point charge evolution equation (23). Importantly, the introduced nonlinearities are stealthy in the sense that they don't show in the dynamics and kinematics of what appears to be soliton-like waves propagating as classical point charges.

Using the relations (78) and (110) we give the following representations for the microcharge, the micro-current and momentum densities

$$
\begin{aligned}
& \rho(t, \mathbf{x})=q \dot{\psi}^{2}(|\mathbf{x}-\mathbf{r}(t)|), \quad \mathbf{J}(t, \mathbf{x})=q \mathbf{v} \dot{\psi}^{2}(|\mathbf{x}-\mathbf{r}(t)|), \\
& \mathbf{P}(t, \mathbf{x})=\frac{m}{q} \mathbf{J}(t, \mathbf{x})=\mathbf{p} \dot{\psi}^{2}(|\mathbf{x}-\mathbf{r}(t)|) .
\end{aligned}
$$

The above expressions and the charge normalization condition (11) readily imply the following representations for the total dressed charge field momentum $\mathrm{P}$ and the total current $\mathrm{J}$ for the solution (19) in terms of point charge quantities, namely

$$
\mathrm{P}=\frac{m}{q} \mathrm{~J}=\int_{\mathbb{R}^{3}} \frac{\chi q}{m} \operatorname{Im} \frac{\nabla \dot{\%}}{\dot{q}} \dot{\psi}^{2} \mathrm{~d} \mathbf{x}=\mathbf{p}=m \mathbf{v} .
$$


We would like to mention that an idea to use concept of a solitary wave in nonlinear dispersive media for modelling wave-particles was quite popular. Luis de Broglie tried to use it in his pursuit of the material wave mechanics. G. Lochak wrote in his preface to the de Broglie's monograph, [13, page XXXIX]: “... The first idea concerns the solitons, which we would call ondes à bosses (humped waves) at the Institut Henri Poincaré. This idea of de Broglie's used to be considered as obsolete and too classical, but it is now quite well known, as I mentioned above, and is likely to be developed in the future, but only provided we realize what the obstacle is and has been for twenty-five years: It resides in the lack of a general principle in the name of which we would be able to choose one nonlinear wave equation from among the infinity of possible equations. If we succeed one day in finding such an equation, a new microphysics will arise." G. Lochak raised an interesting point of the necessity of a general principle that would allow to choose one nonlinearity among infinitely many. We agree to G. Lochak to the extend that there has to be an important physical principle that would allow to choose the nonlinearity but whether it has to be unique is different matter. In our approach such a principle is the exact balance of all forces for the resting dressed charge via the static charge equilibrium equation (15). As to a possibility of spatially localized excitations such as wave-packets to maintain their basic properties when they propagate in a dispersive medium with a nonlinearity we refer to our work [3-5].

Let us derive now the equations of motion (18) for the wave-corpuscle center. If the external field is purely electric and the electric field $\mathbf{E}_{\mathrm{ex}}(t)=-\nabla \varphi_{\mathrm{ex}}(t, \mathbf{x})$ is spatially homogeneous, then the potential $\varphi_{\mathrm{ex}}(t, \mathbf{x})$ is linear in $\mathbf{x}$ and for any given trajectory $\mathbf{r}(t)$ we can write

$$
\varphi_{\mathrm{ex}}(t, \mathbf{x})=\varphi_{0, \mathrm{ex}}(t)+\varphi_{0, \mathrm{ex}}^{\prime}(t) \cdot(\mathbf{x}-\mathbf{r}(t)),
$$

where

$$
\varphi_{0, \mathrm{ex}}^{\prime}(t)=\nabla_{\mathbf{x}} \varphi_{\mathrm{ex}}(\mathbf{r}(t), t) .
$$

The fact that the wave-corpuscle as defined by formula (19) is an exact solution to the field equation (22) can be verified by straightforward examination. Alternatively one can establish the same by considering the expression for $\psi$ in (19) and assuming that the real valued functions $\mathbf{r}(t), \mathbf{v}(t)$ and $s_{\mathrm{p}}(t)$ are unknown and to be found, if possible, from the field equations (22), and that is what we are going to do. Indeed, observe that the representation (19) implies

$$
\partial_{t} \psi=\exp \left(\mathrm{i} \frac{S}{\chi}\right)\left\{\left[\frac{\mathrm{i} m}{\chi}(\dot{\mathbf{v}} \cdot(\mathbf{x}-\mathbf{r})-\mathbf{v} \cdot \dot{\mathbf{r}})+\frac{\mathrm{i} \dot{s}_{\mathrm{p}}}{\chi}\right] \hat{\psi}-\dot{\mathbf{r}} \cdot \nabla \hat{\psi}\right\},
$$

and by the Leibnitz formula we have

$$
\nabla^{2} \psi=\exp \left(\mathrm{i} \frac{S}{\chi}\right)\left[\left(\frac{\mathrm{i} m \mathbf{v}}{\chi}\right)^{2} \hat{\psi}+2 \frac{\mathrm{i} m}{\chi} \mathbf{v} \cdot \nabla \hat{\psi}+\nabla^{2} \hat{\psi}\right] .
$$

To find if the expression (19) for $\psi$ can solve the field equations (22) we substitute the expression into the field equations (22) obtaining the following equation for functions $\mathbf{v}$, r, $s_{\mathrm{p}}$ :

$$
\begin{aligned}
& {\left[-m \dot{\mathbf{v}} \cdot(\mathbf{x}-\mathbf{r})-\mathbf{v} \cdot \dot{\mathbf{r}}-\dot{s}_{\mathrm{p}}\right] \hat{\psi}-\mathrm{i} \chi \dot{\mathbf{r}} \cdot \nabla \hat{\psi}-\frac{m}{2} \mathbf{v}^{2} \hat{\psi}} \\
& \quad+\mathrm{i} \chi \mathbf{v} \cdot \nabla \hat{\psi}+\frac{\chi^{2}}{2 m} \nabla^{2} \hat{\psi}-q\left(\tilde{\varphi}_{\mathrm{ex}}+\varphi\right) \hat{\psi}-\frac{\chi^{2}}{2 m} G^{\prime} \hat{\psi}=0 .
\end{aligned}
$$


Then using the charge equilibrium equation (15) we eliminate the nonlinearity $G$ in the above equation (34) and obtain the following equation equivalent to it:

$$
-\left\{m[\dot{\mathbf{v}} \cdot(\mathbf{x}-\mathbf{r})-\mathbf{v} \cdot \dot{\mathbf{r}}]+\frac{m}{2} \mathbf{v}^{2}+\dot{s}_{\mathrm{p}}+q \tilde{\varphi}_{\mathrm{ex}}\right\} \hat{\psi}-\mathrm{i} \chi(\dot{\mathbf{r}}-\mathbf{v}) \nabla \hat{\psi}=0 .
$$

Now, to determine if there is a triple of functions $\left\{\mathbf{r}(t), \mathbf{v}(t), s_{\mathrm{p}}(t)\right\}$ for which (35) holds, we equate to zero the coefficients before $\nabla \hat{\psi}$ and $\hat{\psi}$ in that equation, resulting in two equations:

$$
\mathbf{v}=\dot{\mathbf{r}}, \quad m[\dot{\mathbf{v}} \cdot(\mathbf{x}-\mathbf{r})-\mathbf{v} \cdot \dot{\mathbf{r}}]+\frac{m}{2} \mathbf{v}^{2}+\dot{s}_{\mathrm{p}}+q \tilde{\varphi}_{\mathrm{ex}}=0,
$$

where, in view of the representation (31), the second equation in (36) can be recast as

$$
m[\dot{\mathbf{v}} \cdot(\mathbf{x}-\mathbf{r})-\mathbf{v} \cdot \dot{\mathbf{r}}]+\dot{s}_{\mathrm{p}}+\frac{m \mathbf{v}^{2}}{2}+q\left[\varphi_{0, \mathrm{ex}}+\varphi_{0, \mathrm{ex}}^{\prime} \cdot(\mathbf{x}-\mathbf{r})\right]=0 .
$$

To find out if there is a triple of functions $\left\{\mathbf{r}(t), \mathbf{v}(t), s_{\mathrm{p}}(t)\right\}$ solving (37) we equate to zero the coefficient before $(\mathbf{x}-\mathbf{r})$ and the remaining coefficient and obtain the following pair of equations

$$
\begin{aligned}
& m \dot{\mathbf{v}}=-q \varphi_{0, \mathrm{ex}}^{\prime}(t), \\
& \dot{s}_{\mathrm{p}}-m \mathbf{v} \cdot \dot{\mathbf{r}}+\frac{m \mathbf{v}^{2}}{2}+q \varphi_{0, \mathrm{ex}}(t)=0 .
\end{aligned}
$$

Thus, based on the first equation (36) and (38), we conclude that the wave-corpuscle defined by the formula (18) with the complementary point charge equations (23), (24) is indeed an exact solution to the field equations (22).

It is instructive to compare our construction of the exact solutions of the form (19) with the quasi-classical approach which uses the WKB ansatz. The trajectories of the charges centers as described by our model coincide with trajectories that can be found by applying well-known quasiclassical asymptotics if one neglects the nonlinearity. Note though that there are two important effects of the nonlinearity not presented in the formal quasiclassical approach. First of all, due to the nonlinearity the charge preserves its shape in the course of evolution whereas in the linear model any wavepacket disperses over time. Second of all, the quasiclassical asymptotic expansions produce infinite asymptotic series which provide for a formal solution, whereas the properly introduced nonlinearity as in (12), (13) allows one to obtain an exact solution. For a treatment of mathematical aspects of the approach to nonlinear wave mechanics based on the WKB asymptotic expansions we refer the reader to [27] and references therein.

Using methods similar to used above let us consider a single charge in a general external EM field which can have nonzero magnetic component. In this case the wave-corpuscle defined by relations (19) and the complementary point charge equations (18), (20) is an exact solution to the auxiliary field equations

$$
\begin{aligned}
\mathrm{i} \chi \partial_{t} \psi & =-\frac{\chi^{2} \nabla^{2} \psi}{2 m}-\frac{\chi q \tilde{\mathbf{A}}_{\mathrm{ex}} \cdot \nabla \psi}{m \mathrm{ci}}+q\left(\varphi+\tilde{\varphi}_{\mathrm{ex}}\right) \psi+\frac{\chi^{2} G^{\prime} \psi}{2 m}, \\
\nabla^{2} \varphi & =-4 \pi q|\psi|^{2}
\end{aligned}
$$

with spatially linear potentials, $\tilde{\varphi}_{\mathrm{ex}}, \tilde{\mathbf{A}}_{\mathrm{ex}}$. Note that in applications to general external fields the linear potential $\tilde{\mathbf{A}}_{\mathrm{ex}}$ is the linearization of $\mathbf{A}_{\mathrm{ex}}(t, \mathbf{x})$ at $\mathbf{r}(t)$ and the potential $\tilde{\varphi}_{\mathrm{ex}}$ is the linearization at $\mathbf{r}(t)$ of the coefficient $\varphi_{\mathrm{ex}}+\frac{q \mathbf{A}_{\mathrm{ex}}^{2} \psi}{2 m \mathrm{c}^{2}}$ in (9). One way to check that $\psi, \varphi$ is a 
solution is just to plug in $\psi, \varphi$ defined by the formulas (19) into the auxiliary field equations (39) and, using the complementary point charge equations (18), (20), to verify that the equality does hold. A derivation of (18), (20) and details are given in [6].

One can naturally ask how broad is the class of external EM fields for which there are exact solutions as the wave-corpuscles? The class of such EM field is sufficiently broad in the sense that for any accelerated motion of a point charge there is wave-corpuscle as an exact solution to the field equations with an external field spatially homogeneous field. To see that let us take an arbitrary vector-function $\mathbf{r}(t)$ and determine linear $\varphi_{\mathrm{ex}}(t, \mathbf{x})$ by formula (31) with $\varphi_{0, \mathrm{ex}}^{\prime}(t)=-m \ddot{\mathbf{r}}(t) / q, \varphi_{0, \mathrm{ex}}=0$ and let $\mathbf{B}_{\mathrm{ex}}=0$. Then $\mathbf{r}(t)$ is a solution of (18) and wave-corpuscle (19) is a solution of (9), (10) with this $\varphi_{\mathrm{ex}}(t, \mathbf{x})$. Thus, we can conclude that the wave-corpuscle (19) as an exact solution to the field equations (9), (10) with an appropriate choice of the external EM field can model any motion of a point charge.

\subsection{Accelerated Motion of a Single Nonrelativistic Charge in a General EM Field}

In the case of a general external EM field no exact closed form solution to the field equations seems to be available, but there is an approximate wave-corpuscle solution and its accuracy is a subject of our studies in this case. For simplicity let us consider purely electric external field. Recall that the wave-corpuscle (19)-(20) is an exact solution to the fields equations (9), (10) if the EM potentials are linear functions of spatial variables. Obviously, that a wavecorpuscle of the form (19), which decays exponentially at large distances, is localized around its center $\mathbf{r}(t)$, with localization radius of order $a$. Therefore, we construct approximate wave-corpuscle solution to the field equations in the case where the potential of the external EM field can be linearized at the location $\mathbf{r}(t)$ with a small error. The error of linearization of the potential can be measured in terms of minimal curvature radius of the graph of the potential, this radius introduces a spatial scale $R_{\varphi}$. Hence, linearization of potentials around the center $\mathbf{r}(t)$ of a wave-corpuscle with size $a$ results in error of order $a^{2} / R_{\varphi}^{2}$. Using this simple observation we define an approximate solution in two steps. First, we determine the trajectories $\mathbf{r}(t)$ as a solution to (20) where $\mathbf{B}_{\mathrm{ex}}=0$. Then we define the wave-corpuscle $\psi(t, \mathbf{x})$ by formulas (19)-(20). It is an exact solution to the auxiliary field equation with the field potential $\tilde{\varphi}_{\mathrm{ex}}$ obtained by linearization of the electric field $\varphi_{\mathrm{ex}}$ at $\mathbf{r}(t)$. Since $\psi(t, \mathbf{x})$ is an exact solution to the auxiliary field equation, and it is localized about $\mathbf{r}(t)$ with localization size $a$, its substitution into field equations (9), (10) produces a discrepancy of order $a^{2} / R^{2}$. Hence, the condition for an integral discrepancy to be relatively small takes the form

$$
\frac{a^{2}}{R_{\varphi}^{2}} \ll 1 .
$$

One can use similar to the above treatment in the case when the external magnetic field is present but estimates are more involved. In that case, if we introduce a spatial scale $R_{M}$ at which magnetic field varies significantly then the conditions for an integral discrepancy to be relatively small takes the form

$$
\frac{|\mathbf{v}|}{\mathrm{c}} \frac{a}{R_{M}} \ll 1, \quad \frac{a^{2}}{R_{\varphi}^{2}} \ll 1,
$$

where $\mathbf{v}$ is the velocity of the wave-corpuscle center. 


\subsection{Nonlinear Self-Interaction, Its Basic Properties and Examples}

As we have already explained in the beginning of Sect. 2 the nonlinear self interaction function $G$ is determined from the charge equilibrium equation (15) based on the form factor $\dot{\psi}$ and the form factor potential $\stackrel{\circ}{\varphi}$. It is worth to point out that such a nonlinearity differs significantly from nonlinearities considered in similar problems in literature. Important features of our nonlinearity include: (i) the boundedness of its derivative $G^{\prime}(s)$ for $s \geq 0$ with consequent boundedness from below of the wave energy; (ii) non analytic behavior about $s=0$, that is for small wave amplitudes.

In this section we consider the construction of the function $G$, study its properties and provide examples for which the construction of $G$ is carried out explicitly. Throughout this section we have

$$
\psi, \dot{\psi} \geq 0 \text { and hence }|\psi|=\psi .
$$

We introduce explicitly the size parameter $a>0$ through the following representation of the fundamental functions $\stackrel{\leftrightarrow}{\psi}(r)$ and $\stackrel{\circ}{\varphi}(r)$

$$
\begin{aligned}
& \stackrel{\leftrightarrow}{\psi}(r)=\dot{\psi}_{a}(r)=a^{-3 / 2} \dot{\psi}_{1}\left(a^{-1} r\right), \\
& \stackrel{\varphi}{\varphi}(r)=\dot{\varphi}_{a}(r)=a^{-1} \stackrel{\circ}{\varphi}_{1}\left(a^{-1} r\right),
\end{aligned}
$$

where $\dot{\psi}_{1}(r)$ and $\stackrel{\circ}{\varphi}_{1}(r)$ are functions of the dimensionless parameter $r$, and, as a consequence of (80), the function $\dot{\psi}_{a}(r)$ satisfies the charge normalization condition

$$
\int_{\mathbb{R}^{3}} \stackrel{\circ}{\psi}_{a}^{2}(|\mathbf{x}|) \mathrm{d} \mathbf{x}=1 \quad \text { for all } a>0
$$

The size parameter a naturally has the dimension of length, but we do not yet identify it with the size. Indeed, any properly defined spatial size of $\dot{\psi}_{a}$, based, for instance, on the variance, is proportional to $a$ with a coefficient depending on $\dot{\psi}_{1}$. The charge equilibrium equation (15) can be written in the following form:

$$
-\frac{\chi^{2}}{2 m} \nabla^{2} \dot{\psi}_{a}+q \dot{\varphi}_{a} \dot{\psi}_{a}+\frac{\chi^{2}}{2 m} G_{a}^{\prime}\left(\dot{\psi}_{a}^{2}\right) \dot{\psi}_{a}=0,
$$

where the potential $\dot{\varphi}_{a}$ is defined by (14). The function $\dot{\psi}_{a}(r)$ is assumed to be a positive, monotonically decreasing function of $r \geq 0$, and to satisfy the charge normalization condition (43).

Let us look first at the case $a=1, \stackrel{\leftrightarrow}{\psi}_{a}=\stackrel{\leftrightarrow}{\psi}_{1}, \stackrel{\circ}{\varphi}_{a}=\stackrel{\circ}{\varphi}_{1}$, for which (44) yields the following representation for $G^{\prime}\left(\dot{\psi}_{1}^{2}\right)$ from (44)

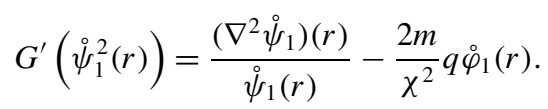

Since $\AA_{1}^{2}(r)$ is a monotonic function, we can find its inverse $r=r\left(\psi^{2}\right)$, yielding

$$
\begin{aligned}
G^{\prime}(s) & =\left[\frac{\nabla^{2} \stackrel{\circ}{1}_{1}}{\dot{\vartheta}_{1}}-\frac{2 m}{\chi^{2}} q \stackrel{\circ}{1}_{1}\right](r(s)), \\
0 & =\dot{\psi}_{1}^{2}(\infty) \leq s \leq \dot{\psi}_{1}^{2}(0) .
\end{aligned}
$$


We extend $G^{\prime}(s)$ for $s \geq \dot{\psi}_{1}^{2}(0)$ to be a constant, namely

$$
G^{\prime}(s)=G^{\prime}\left(\dot{\psi}_{1}^{2}(0)\right) \quad \text { if } s \geq \dot{\psi}_{1}^{2}(0) .
$$

Observe that the positivity and the monotonicity of the form factor $\dot{\psi}_{1}$ was instrumental for recovering the function $G^{\prime}(s)$ from the charge balance equation (44).

Using the representation (46) for the function $G^{\prime}(s)$ we decompose it naturally into two components:

$$
G^{\prime}(s)=G_{\nabla}^{\prime}(s)-\frac{2}{a_{\chi}} G_{\varphi}^{\prime}(s),
$$

where

$$
a_{\chi}=\frac{\chi^{2}}{m q^{2}}
$$

and for all $r \geq 0$

$$
\begin{aligned}
& G_{\nabla}^{\prime}\left(\stackrel{\circ}{\psi}_{1}^{2}\right)(r)=\frac{\left(\nabla^{2} \dot{\leftrightarrow}_{1}\right)}{\stackrel{\circ}{\psi}_{1}}(r), \\
& G_{\varphi}^{\prime}\left(\stackrel{\circ}{\psi}_{1}^{2}\right)(r)=\frac{\stackrel{\circ}{\varphi}_{1}(r)}{q}=\stackrel{\circ}{\phi}_{1}(r) .
\end{aligned}
$$

We refer to $G_{\nabla}^{\prime}(s)$ and $G_{\varphi}^{\prime}(s)$ as elastic and EM components respectively. In the case of arbitrary size parameter $a$ we find first that

$$
\begin{aligned}
G_{\nabla, a}^{\prime}(s) & =a^{-2} G_{\nabla, 1}^{\prime}\left(a^{3} s\right), \\
G_{\varphi, a}^{\prime}(s) & =a^{-1} G_{\varphi, 1}^{\prime}\left(a^{3} s\right), \quad a>0,
\end{aligned}
$$

and then combining (51) with (48) and (50) we obtain the following representation for the function $G_{a}^{\prime}(s)$

$$
G_{a}^{\prime}(s)=\frac{G_{\nabla, 1}^{\prime}\left(a^{3} s\right)}{a^{2}}-\frac{2 G_{\varphi, 1}^{\prime}\left(a^{3} s\right)}{a a_{\chi}} .
$$

Let us take a look at general properties of $G^{\prime}(s)$ and its components $G_{\nabla}^{\prime}(s)$ and $G_{\varphi}^{\prime}(s)$ as they follow from defining them relations (46)-(52). Starting with the EM component $G_{\varphi}^{\prime}(s)$ we notice that $\stackrel{\varphi}{1}_{1}(|\mathbf{x}|)$ is a radial solution to (10). Combining that with $\psi^{2} \geq 0$ and using the Maximum principle we conclude that $\stackrel{\circ}{1}_{1}(|\mathbf{x}|) / q$ is a positive function without local minima, implying that it is a monotonically decreasing function of $|\mathbf{x}|$. Consequently, $G_{\varphi}^{\prime}(s)$ defined by (50) is a monotonically increasing function of $s$, and hence

$$
G_{\varphi}^{\prime}(s)>0 \quad \text { for all } s>0 \text { and } \quad G_{\varphi}^{\prime}(0)=0 .
$$

Note that $G_{\varphi}^{\prime}(s)$ is not differentiable at zero, which can be seen by comparing the behavior of $\stackrel{\circ}{\varphi}_{1}(r)$ and $\dot{\psi}_{1}(r)$ at infinity. Indeed, $\varphi_{1}(r) / q \sim r^{-1}$ as $r \rightarrow \infty$ and since $\dot{\psi}^{2}(|\mathbf{x}|)$ is integrable, it has to decay faster than $|\mathbf{x}|^{-3}$ as $|\mathbf{x}| \rightarrow \infty$. Consequently, $\left|G_{\varphi}^{\prime}(s)\right|$ for small $s$ has to be greater than $s^{1 / 3}$ which prohibits its differentiability at zero. One has to notice though that the nonlinearity $G^{\prime}\left(|\psi|^{2}\right) \psi$ as it enters the field equation (9)-(10) is differentiable for all $\psi$ including zero, hence it satisfies a Lipschitz condition required for uniqueness of solutions of the initial value problem for (9)-(10). 
Let us look at the elastic component $G_{\nabla}^{\prime}(s)$ defined by the relations (50). Since $\stackrel{\AA}{\psi}(|\mathbf{x}|)>0$ the sign of $G_{\nabla}^{\prime}\left(|\psi|^{2}\right)$ coincides with the sign of $\nabla^{2} \dot{\psi}_{1}(|\mathbf{x}|)$. At the origin $\mathbf{x}=\mathbf{0}$ the function $\stackrel{\varkappa}{1}_{1}(|\mathbf{x}|)$ has its maximum and, consequently, $G_{\nabla}^{\prime}(s) \leq 0$ for all $s$ close to $s=\dot{\psi}_{1}^{2}(\infty)$, implying

$$
G_{\nabla}^{\prime}(s) \leq 0 \text { for } s \gg 1 .
$$

The Laplacian applied to radial functions $\dot{\psi}_{1}$ takes the form $\frac{1}{r} \frac{\partial^{2}}{\partial r^{2}}\left(r \stackrel{\circ}{1}_{1}|\mathbf{x}|\right)$. Consequently, if $r \dot{\psi}_{1}(r)$ is convex at $r=|\mathbf{x}|$ we have $\nabla^{2} \stackrel{\leftrightarrow}{\psi}_{1}(|\mathbf{x}|) \geq 0$. Since $r^{2} \stackrel{\circ}{\psi}(r)$ is integrable we can naturally assume that $|\mathbf{x}| \dot{\psi}_{1}(|\mathbf{x}|) \rightarrow 0$ as $|\mathbf{x}| \rightarrow \infty$. Then if the second derivative of $r \dot{\psi}_{1}(r)$ has a constant sign near infinity, it must be non-negative. For an exponentially decaying $\stackrel{\circ}{\psi}_{1}(r)$ the second derivative of $r \dot{\psi}_{a}(r)$ is positive implying

$$
G_{\nabla}^{\prime}(s)>0 \text { for } s \ll 1 .
$$

Combining this with the equality $G_{\varphi}^{\prime}(0)=0$ from (53) we readily obtain

$$
G^{\prime}(s)>0 \text { for } s \ll 1 \text {. }
$$

From the relations (48), (53), (54) we also obtain

$$
G^{\prime}(s)<0 \text { if } s \gg 1 \text {. }
$$

\subsubsection{Nonlinearity for the Form Factor Decaying as a Power Law}

We introduce here a form factor $\dot{\psi}_{1}(r)$ decaying as a power law of the form

$$
\stackrel{\circ}{\psi}_{1}(r)=\frac{c_{\mathrm{pw}}}{\left(1+r^{2}\right)^{5 / 4}},
$$

where $c_{\mathrm{pw}}$ is the normalization factor, $c_{\mathrm{pw}}=3^{1 / 2} /(4 \pi)^{1 / 2}$. This function evidently is positive and monotonically decreasing as required. Let us find now $G_{\nabla}^{\prime}(s)$ and $G_{\varphi}^{\prime}$ based on the relations (50). An elementary computation of $\nabla^{2} \stackrel{\circ}{\psi}_{1}$ shows that

$$
\begin{aligned}
G_{\nabla}^{\prime}(s) & =\frac{15 s^{2 / 5}}{4 c_{\mathrm{pw}}^{4 / 5}}-\frac{45 s^{4 / 5}}{4 c_{\mathrm{pw}}^{8 / 5}}, \\
G_{\nabla}(s) & =\frac{75 s^{7 / 5}}{28 c_{\mathrm{pw}}^{4 / 5}}-\frac{25 s^{9 / 5}}{4 c_{\mathrm{pw}}^{8 / 5}}, \quad \text { for } 0 \leq s \leq c_{\mathrm{pw}}^{2} .
\end{aligned}
$$

To determine $G_{\varphi}^{\prime}$ we find by a straightforward examination that function

$$
\stackrel{\circ}{\varphi}_{1}=\frac{q}{c_{\mathrm{pw}}^{2 / 5}} \stackrel{\leftrightarrow}{\psi}_{1}^{2 / 5}
$$

solves equation $\nabla^{2} \stackrel{\circ}{1}_{1}=-4 \pi q \dot{\psi}_{1}^{2}$, and that together with (50) yields

$$
G_{\varphi}^{\prime}(s)=\frac{s^{1 / 5}}{c_{\mathrm{pw}}^{2 / 5}}, \quad G_{\varphi}(s)=\frac{5 s^{6 / 5}}{6 c_{\mathrm{pw}}^{2 / 5}}, \quad \text { for } 0 \leq s \leq c_{\mathrm{pw}}^{2} .
$$

Observe that both components $G_{\nabla}^{\prime}(s)$ and $G_{\varphi}^{\prime}(s)$ in (59), (61) of the total nonlinearity $G_{\nabla}^{\prime}(s)$ defined by (48) are not differentiable at $s=0$. 
If we explicitly introduce size parameter $a$ into the form factor using (42), we define $G_{\nabla, a}^{\prime}(s)$ and $G_{\varphi, a}^{\prime}(s)$ and $G_{a}^{\prime}(s)$ by (51) and (52). Notice that the variance of the form factor $\stackrel{\circ}{1}_{1}^{2}(|\mathbf{x}|)$ decaying as a power law (58) is infinite.

\subsubsection{Nonlinearity for the Form Factor Decaying Exponentially}

We introduce here an exponentially decaying form factor $\dot{\psi}_{1}$ of the form

$$
\stackrel{\circ}{\psi}_{1}(r)=c_{\mathrm{e}} \mathrm{e}^{-\left(r^{2}+1\right)^{1 / 2}},
$$

where $c_{\mathrm{e}}$ is the normalization factor,

$$
c_{\mathrm{e}}=\left(4 \pi \int_{0}^{\infty} r^{2} \mathrm{e}^{-2\left(r^{2}+1\right)^{1 / 2}} \mathrm{~d} r\right)^{-1 / 2} \simeq 0.79195 .
$$

Evidently $\dot{\psi}_{1}(r)$ is positive and monotonically decreasing as required. The dependence $r(s)$ defined by the relation (62) is as follows:

$$
r=\left[\ln ^{2}\left(c_{\mathrm{e}} / \sqrt{s}\right)-1\right]^{1 / 2}, \quad \text { if } \sqrt{s} \leq \dot{\psi}_{1}(0)=c_{\mathrm{e}} \mathrm{e}^{-1} .
$$

An elementary computation shows that $\nabla^{2} \dot{\psi}_{1}=-W \dot{\psi}_{1}$ where

$$
W=\frac{2}{\left(r^{2}+1\right)^{\frac{1}{2}}}+\frac{1}{\left(r^{2}+1\right)}+\frac{1}{\left(r^{2}+1\right)^{\frac{3}{2}}}-1,
$$

implying

$$
G_{\nabla}^{\prime}\left(\psi_{1}^{2}(r)\right)=-W(r)=1-\frac{2}{\left(r^{2}+1\right)^{\frac{1}{2}}}-\frac{1}{\left(r^{2}+1\right)}-\frac{1}{\left(r^{2}+1\right)^{\frac{3}{2}}} .
$$

Combining (63) with (64) we readily obtain the following function for $s \leq c_{\mathrm{e}}^{2} \mathrm{e}^{-2}$

$$
G_{\nabla, 1}^{\prime}(s)=\left[1-\frac{4}{\ln \left(c_{\mathrm{e}}^{2} / s\right)}-\frac{4}{\ln ^{2}\left(c_{\mathrm{e}}^{2} / s\right)}-\frac{8}{\ln ^{3}\left(c_{\mathrm{e}}^{2} / s\right)}\right] .
$$

We extend it for larger $s$ as follows:

$$
G_{\nabla, 1}^{\prime}(s)=G_{\nabla, 1}^{\prime}\left(c_{\mathrm{e}}^{2} \mathrm{e}^{-2}\right)=-3 \quad \text { if } s \geq c_{\mathrm{e}}^{2} \mathrm{e}^{-2} .
$$

The relations (65) and (66) imply $G_{\nabla, 1}^{\prime}(s)$ takes values in the interval [1, -3]. It also follows from (65) that

$$
G_{\nabla, 1}^{\prime}(s) \cong 1-\frac{4}{\ln 1 / s} \quad \text { as } s \rightarrow 0,
$$

implying that the function $G_{\nabla, 1}^{\prime}(s)$ is not differentiable at $s=0$ and consequently is not analytic about 0 .

To determine the second component $G_{\varphi}^{\prime}$ we need to solve (50). Using the fact that $\stackrel{\circ}{\varphi}_{1}, \stackrel{\leftrightarrow}{\psi}_{1}$ are radial functions we obtain the following equation for $\stackrel{\circ}{\varphi}_{1}(r)$

$$
-\frac{1}{4 \pi r} \partial_{r}^{2}\left(r \stackrel{\circ}{1}_{1}\right)=q c_{\mathrm{e}}^{2} \mathrm{e}^{-2 \sqrt{r^{2}+1}}
$$


We seek a solution of (68) that is regular at zero and behaves like the Coulomb's potential $\frac{q}{r}$ for large $r$. Taking that into account we obtain after the first integration of (68)

$$
\partial_{r}\left(r \stackrel{\circ}{\varphi}_{1}\right)=\pi q c_{\mathrm{e}}^{2}\left[1+2\left(r^{2}+1\right)^{1 / 2}\right] \mathrm{e}^{-2\left(r^{2}+1\right)^{1 / 2}},
$$

and integrating (69) yields the following formula for the form factor potential:

$$
\stackrel{\circ}{\varphi}_{1}(r)=\frac{q}{r}-\frac{\pi q c_{\mathrm{e}}^{2}}{r} \int_{r}^{\infty}\left[1+2\left(r_{1}^{2}+1\right)^{1 / 2}\right] \mathrm{e}^{-2\left(r_{1}^{2}+1\right)^{1 / 2}} \mathrm{~d} r_{1} .
$$

The above formula shows that the form factor potential $\stackrel{\circ}{\varphi}_{1}(r)$ is exponentially close to the Coulomb's potential $q / r$ for large $r$. Then, based on the relation (50) and (70), we find consequently

$$
G_{\varphi, 1}^{\prime}\left(\psi^{2}\right)=\frac{1}{q} \stackrel{\circ}{\varphi}_{1}(r(\psi))=\frac{\pi c_{\mathrm{e}}^{2}}{r(\psi)} \int_{0}^{r(\psi)}\left[1+2\left(r_{1}^{2}+1\right)^{1 / 2}\right] \mathrm{e}^{-2\left(r_{1}^{2}+1\right)^{1 / 2}} \mathrm{~d} r_{1},
$$

where

$$
r(\psi)=\left[\ln ^{2}\left(c_{\mathrm{e}} / \psi\right)-1\right]^{1 / 2} \text { for } \psi \leq c_{\mathrm{e}} \mathrm{e}^{-1}
$$

We extend $G_{\varphi, 1}^{\prime}\left(\psi^{2}\right)$ for larger values of $\psi$ as a constant:

$$
G_{\varphi, 1}^{\prime}\left(\psi^{2}\right)=\lim _{r \rightarrow 0} \frac{\stackrel{\circ}{\varphi}(r)}{q}=3 \pi c_{\mathrm{e}}^{2} \mathrm{e}^{-2} \text { for } \psi \geq c_{\mathrm{e}} \mathrm{e}^{-1} .
$$

From the above expressions one can see that $G_{a}^{\prime}(s)$ does not depend analytically on $s$ about $s=0$. The variance of the exponential form factor $\dot{\psi}_{1}(r)$ is obviously finite. To find $G_{a}^{\prime}(s)$ for arbitrary $a$ we use its representation (52), and combine with the formulas (65) and (70).

\subsection{Form Factor Potential Proximity to the Coulomb's Potential}

In this section we study the proximity of the potential form factor $\dot{\varphi}_{a}(|\mathbf{x}|)$ to the Coulomb's potential $q /|\mathbf{x}|$ for small $a$. This is an important issue since it is a well known experimental fact that the Coulomb's potential $q /|\mathbf{x}|$ represents the electrostatic field of the charge very accurately even for very small values of $|\mathbf{x}|$. According to the rest charge equation (15) and (14) the potential $\stackrel{\circ}{\phi}_{a}(|\mathbf{x}|)=\stackrel{\circ}{\varphi}_{a}(|\mathbf{x}|) / q$ satisfies

$$
\nabla^{2} \stackrel{\phi}{a}_{a}(|\mathbf{x}|)=-4 \pi \stackrel{\leftrightarrow}{q}_{a}^{2}(|\mathbf{x}|) \text {. }
$$

For the radial potential $\phi_{1}(r)$ we obtain, [6],

$$
\phi_{1}(r)=\frac{1}{r}\left[1-4 \pi \int_{r}^{\infty}\left(r_{1}-r\right) r_{1} \dot{\psi}_{1}^{2}\left(r_{1}\right) \mathrm{d} r_{1}\right] .
$$

Formula (73) together with (42) implies that

$$
\phi_{a}(r)=\frac{1}{r}-\frac{4 \pi}{r} \int_{a^{-1} r}^{\infty}\left(r_{1}-a^{-1} r\right) r_{1} \dot{\psi}_{1}^{2}\left(r_{1}\right) \mathrm{d} r_{1} .
$$

We notice that if $\dot{\psi}_{1}^{2}$ decays exponentially as in (62) then the difference between the potential $\phi_{a}(r)$ and the Coulomb's potential $1 / r$ is extremely small for small $a$. Notice that if we 
would take $\dot{\psi}_{1}(r)=0$ for all $r \geq \mathrm{r}_{0}$, as it is the case in the Abraham-Lorentz model, the formula (73) would imply that $\dot{\varphi}_{a}(r)$ would be exactly the Coulomb's potential for $r \geq$ $a \mathrm{r}_{0}$. But for such a $\dot{\psi}_{1}(r)$ we would not be able to construct the nonlinear self-interaction component $G_{\varphi}^{\prime}$ which would satisfy (50) since it requires $\dot{\psi}_{1}^{2}(r)$ to be strictly positive for all $r \geq 0$.

\subsection{Many Interacting Charges}

A qualitatively new physical phenomenon in the theory of two or more charges compared with the theory of a single charge is obviously the interaction between them. In our approach any individual "bare" charge interacts directly only with the EM field and consequently different charges interact with each other only indirectly through the EM field. A system of many charges can have charges of different type, for instance electrons and protons. In that case we naturally assume that individual Lagrangians for charges of the same type have identical Lagrangians with the same mass $m$, charge $q$, form factor $\dot{\psi}$ and consequently the same nonlinear self-interaction $G$. Below we provide a sketch of the theory and more detailed exposition of the subject is provided in our manuscript [6].

The primary focus of our studies on many charges here the correspondence between our wave theory and the point charge mechanics in the regime of remote interaction when the charges are separated by large distances compared to their sizes. There are two distinct ways to correspond our field theory to the point charge mechanics: (i) via averaged quantities in spirit of the well known in quantum mechanics Ehrenfest Theorem, [38, Sects. 7, 23]; (ii) via a construction of approximate solutions to the field equations in terms of radial wavecorpuscles similar to (19).

Studies of charge interactions at short distances are not within the scope of this paper, though our approach allows to study short distance interactions and we provide as an example the hydrogen atom model in Sect. 4.1. The main purpose of that exercise is to show a similarity between our and Schrödinger's hydrogen atom models, and to contrast it to any kind of Kepler's model. Another point we can make based on our hydrogen atom model is that our theory does provide a basis for a regime of close interaction between two charges which differs significantly from the regime of remote interaction, which is the primary focus of this paper.

Let us consider a system of $N$ charges interacting directly only with the EM field described by its 4 -vector potential $A^{\mu}=(\varphi, \mathbf{A})$. The charges are described by their wave functions $\psi^{\ell}$ with the superscript index $\ell=1, \ldots, N$ labeling them. Our nonrelativistic Lagrangian $\hat{\mathcal{L}}$ for many charges is constructed based on the following principles: (i) individual charges nonrelativistic Lagrangians $\hat{L}^{\ell}$ of the form (7) and (ii) the assumption that every charge interacts directly only with the EM field as defined by its electric potential $\varphi$. The form on the Lagrangian $\hat{\mathcal{L}}$ in the presence of an external EM field with potentials $\varphi_{\mathrm{ex}}(t, \mathbf{x})$, $\mathbf{A}_{\mathrm{ex}}(t, \mathbf{x})$ is as follows

$$
\begin{aligned}
& \hat{\mathcal{L}}=\sum_{\ell=1}^{N} \hat{L}^{\ell}+\frac{|\nabla \varphi|^{2}}{8 \pi}, \\
& \hat{L}^{\ell}=\frac{\chi}{2} \mathrm{i}\left[\psi^{\ell *} \tilde{\partial}_{t}^{\ell} \psi^{\ell}-\psi^{\ell} \tilde{\partial}_{t}^{\ell *} \psi^{\ell *}\right]-\frac{\chi^{2}}{2 m}\left[\tilde{\nabla} \psi^{\ell} \cdot \tilde{\nabla}^{*} \psi^{\ell *}+G_{a}^{\ell}\left(\psi^{\ell *} \psi^{\ell}\right)\right], \\
& \tilde{\partial}_{t}^{\ell}=\partial_{t}+\frac{\mathrm{i} q^{\ell}\left(\varphi+\varphi_{\mathrm{ex}}\right)}{\chi}
\end{aligned}
$$


where $\psi^{\ell *}$ is complex conjugate of $\psi^{\ell}$ and the covariant gradient $\tilde{\nabla}$ is the same as in (8). The nonlinear self-interaction terms $G_{a}^{\ell}$ in (75) are determined through the charge equilibrium equation (15) and they can be the same for the same types of charges, for instance, protons and electrons. We also indicated in the notation $G_{a}^{\ell}$ the dependence on the size parameter $a$. The field equations for this Lagrangian are

$$
\begin{aligned}
\mathrm{i} \chi \partial_{t} \psi^{\ell}= & -\frac{\chi^{2} \nabla^{2} \psi^{\ell}}{2 m^{\ell}}-\frac{\chi q^{\ell} \mathbf{A}_{\mathrm{ex}} \cdot \nabla \psi^{\ell}}{m^{\ell} \mathrm{c} i} \\
& +q^{\ell}\left(\varphi+\varphi_{\mathrm{ex}}\right) \psi^{\ell}+\frac{q^{\ell 2} \mathbf{A}_{\mathrm{ex}}^{2} \psi^{\ell}}{2 m^{\ell} \mathrm{c}^{2}}+\left[G_{a}^{\ell}\right]^{\prime}\left(\left|\psi^{\ell}\right|^{2}\right) \psi^{\ell}, \\
-\nabla^{2} \varphi= & 4 \pi \sum_{\ell=1}^{N} q^{\ell}\left|\psi^{\ell}\right|^{2} .
\end{aligned}
$$

Evidently the equations for different $\ell$ are coupled only through the potential $\varphi$, which consequently is responsible for the charge interaction. The Lagrangian $\hat{\mathcal{L}}$ is gauge invariant and every $\ell$-th charge has a 4-current $\left(\rho^{\ell}, \mathbf{J}^{\ell}\right)$ defined by

$$
\rho^{\ell}=q\left|\psi^{\ell}\right|^{2}, \quad \mathbf{J}^{\ell}=\left(\frac{\chi q^{\ell}}{m^{\ell}} \operatorname{Im} \frac{\nabla \psi^{\ell}}{\psi^{\ell}}-\frac{q^{\ell 2} \mathbf{A}_{\mathrm{ex}}}{m^{\ell} \mathrm{c}}\right)\left|\psi^{\ell}\right|^{2},
$$

satisfying the conservation/continuity equations $\partial_{t} \rho^{\ell}+\nabla \cdot \mathbf{J}^{\ell}=0$ or explicitly

$$
m^{\ell} \partial_{t}\left|\psi^{\ell}\right|^{2}+\nabla \cdot\left(\chi \operatorname{Im} \frac{\nabla \psi^{\ell}}{\psi^{\ell}}\left|\psi^{\ell}\right|^{2}-\frac{q^{\ell}}{\mathrm{c}} \mathbf{A}_{\mathrm{ex}}\left|\psi^{\ell}\right|^{2}\right)=0 .
$$

In consistency with the above charge conservation equation we require the $\ell$-th charge to be exactly $q^{\ell}$ by imposing the following charge normalization condition:

$$
\int_{\mathbb{R}^{3}}\left|\psi^{\ell}\right|^{2} \mathrm{~d} \mathbf{x}=1, \quad t \geq 0, \ell=1, \ldots, N
$$

Let us attribute to every $\ell$-th charge its potential $\varphi^{\ell}$ by the formula

$$
\varphi^{\ell}(t, \mathbf{x})=\varphi_{a}^{\ell}(t, \mathbf{x})=q^{\ell} \int_{\mathbb{R}^{3}} \frac{\left|\psi_{a}^{\ell}\right|^{2}(t, \mathbf{y})}{|\mathbf{y}-\mathbf{x}|} \mathrm{d} \mathbf{y},
$$

implying together with (77) the following relations

$$
\nabla^{2} \varphi^{\ell}=-4 \pi q^{\ell}\left|\psi^{\ell}\right|^{2}, \quad \varphi=\sum_{\ell=1}^{N} \varphi^{\ell} .
$$

We also introduce a potential $\varphi_{\mathrm{ex}, a}^{\ell}$ describing the interaction of $\ell$-th charge with all other charges:

$$
\varphi_{\mathrm{ex}, a}^{\ell}=\varphi+\varphi_{\mathrm{ex}}-\varphi_{a}^{\ell}=\varphi_{\mathrm{ex}}+\sum_{\ell^{\prime} \neq \ell} \varphi_{a}^{\ell^{\prime}} .
$$

The charge gauge invariant momentum density $\mathbf{P}^{\ell}$ for the Lagrangian $\hat{\mathcal{L}}$ is of the form

$$
\mathbf{P}^{\ell}=\frac{\mathrm{i} \chi}{2}\left(\psi^{\ell} \tilde{\nabla}^{\ell *} \psi^{\ell *}-\psi^{\ell *} \tilde{\nabla}^{\ell} \psi^{\ell}\right)
$$


Introducing the total individual momenta $\mathrm{P}^{\ell}$ for $\ell$-th dressed charge by

$$
\mathrm{P}^{\ell}=\int_{\mathbb{R}^{3}} \mathbf{P}^{\ell} \mathrm{d} \mathbf{x}
$$

we obtain the following equations for the total individual momenta

$$
\begin{aligned}
\frac{\mathrm{d} \mathrm{P}^{\ell}}{\mathrm{d} t} & =q^{\ell} \int_{\mathbb{R}^{3}}\left[\left(\sum_{\ell^{\prime} \neq \ell} \mathbf{E}^{\ell^{\prime}}+\mathbf{E}_{\mathrm{ex}}\right)\left|\psi^{\ell}\right|^{2}+\frac{1}{\mathrm{c}} \mathbf{v}^{\ell} \times \mathbf{B}_{\mathrm{ex}}\right] \mathrm{d} \mathbf{x}, \\
\mathbf{v}^{\ell}(t, \mathbf{x}) & =\frac{1}{m^{\ell}} \mathbf{P}^{\ell}(t, \mathbf{x})=\frac{1}{q^{\ell}} \mathbf{J}^{\ell}(t, \mathbf{x}) \quad \text { is the velocity density. }
\end{aligned}
$$

The external EM fields $\mathbf{E}_{\mathrm{ex}}, \mathbf{B}_{\mathrm{ex}}$ in (85) corresponding to the potentials $\varphi_{\mathrm{ex}}, \mathbf{A}_{\mathrm{ex}}$ are determined by standard formulas (131). Derivation of (85) is rather elementary. Indeed, in the simplest case where $\mathbf{A}_{\mathrm{ex}}=0$ we multiply (77) by $\nabla \psi^{\ell *}$, take the real part and integrate the result over the entire space using integration by parts and equalities (82) multiplied by $\nabla \psi^{\ell *}$ to eliminate term with $\ell^{\prime}=\ell$. To obtain (85) in more involved general case one can similarly multiply (77) by $\tilde{\nabla}^{\ell *} \psi^{\ell *}$ and then integrate the result using some vector algebra manipulation; a derivation based on general properties of the energy-momentum tensor is given in [6].

Observe an important property of the Lagrangian $\hat{\mathcal{L}}$ : the charge gauge invariant momentum density $\mathbf{P}^{\ell}$ equals exactly the microcurrent density $\mathbf{J}^{\ell}$ defined by (78) multiplied by the constant $m^{\ell} / q^{\ell}$, namely:

$$
\mathbf{P}^{\ell}=\frac{m^{\ell}}{q^{\ell}} \mathbf{J}^{\ell}
$$

that can be viewed as the momentum density kinematic representation.

\subsubsection{Newtonian Mechanics as an Approximation}

Let us show now that if the size parameter is small compared to the typical scale of variation of EM fields the charge evolution can be described approximately by Newton equations with Lorentz forces. For that purpose we introduce the $\ell$-th charge position $\mathbf{r}^{\ell}(t)$ and velocity $v^{\ell}(t)$ as the following spatial averages

$$
\begin{aligned}
& \mathbf{r}^{\ell}(t)=\mathbf{r}_{a}^{\ell}(t)=\int_{\mathbb{R}^{3}} \mathbf{x}\left|\psi_{a}^{\ell}(t, \mathbf{x})\right|^{2} \mathrm{~d} \mathbf{x}, \\
& \mathbf{v}^{\ell}(t)=\frac{1}{q^{\ell}} \int_{\mathbb{R}^{3}} \mathbf{J}^{\ell}(t, \mathbf{x}) \mathrm{d} \mathbf{x} .
\end{aligned}
$$

Then one can show that a combination of the continuity equation (79) with the momentum evolution equations (85) imply the following remarkable property: the positions $\mathbf{r}^{\ell}(t)$ satisfy with a high accuracy Newton's equations of motion for the system of $N$ point charges if the size parameter $a$ is small enough compared with a typical spatial scale $R_{\mathrm{EM}}$ of the system of point charges. Indeed, using the charge conservation law (79) we find the following identities

$$
\frac{\mathrm{d} \mathbf{r}^{\ell}(t)}{\mathrm{d} t}=\int_{\mathbb{R}^{3}} \mathbf{x} \partial_{t}\left|\psi^{\ell}\right|^{2} \mathrm{~d} \mathbf{x}=-\frac{1}{q^{\ell}} \int_{\mathbb{R}^{3}} \mathbf{x} \nabla \cdot \mathbf{J}^{\ell} \mathrm{d} \mathbf{x}=\frac{1}{q^{\ell}} \int_{\mathbb{R}^{3}} \mathbf{J}^{\ell} \mathrm{d} \mathbf{x}=\mathrm{v}^{\ell}(t),
$$


showing the positions and velocities defined by formulas (88) are related exactly as in the point charge mechanics. Then integrating the momentum density kinematic representation (87) we obtain the following kinematic representation for the dressed charge total momentum

$$
\mathrm{P}^{\ell}(t)=\frac{m^{\ell}}{q^{\ell}} \int_{\mathbb{R}^{3}} \mathbf{J}^{\ell}(t, \mathbf{x}) \mathrm{d} \mathbf{x}=m^{\ell} \mathbf{v}^{\ell}(t),
$$

which also is exactly the same as for point charges mechanics. Relations (89) and (90) yield

$$
m^{\ell} \frac{\mathrm{d}^{2} \mathbf{r}^{\ell}(t)}{\mathrm{d}^{2} t}=m^{\ell} \frac{\mathrm{d}}{\mathrm{d} t} \mathrm{v}^{\ell}(t)=\frac{\mathrm{d} \mathrm{P}^{\ell}}{\mathrm{d} t},
$$

and we obtain from (85) the following system of equations of motion for $N$ charges:

$$
m^{\ell} \frac{\mathrm{d}^{2} \mathbf{r}^{\ell}(t)}{\mathrm{d}^{2} t}=q^{\ell} \int_{\mathbb{R}^{3}}\left[\left(\sum_{\ell^{\prime} \neq \ell} \mathbf{E}^{\ell^{\prime}}+\mathbf{E}_{\mathrm{ex}}\right)\left|\psi^{\ell}\right|^{2}+\frac{1}{\mathrm{c}} \mathbf{v}^{\ell} \times \mathbf{B}_{\mathrm{ex}}\right] \mathrm{d} \mathbf{x},
$$

where $\ell=1, \ldots, N$, and $\mathbf{E}^{\ell^{\prime}}(t, \mathbf{x})=-\nabla \varphi^{\ell^{\prime}}(t, \mathbf{x}), \mathbf{E}_{\mathrm{ex}}$ and $\mathbf{B}_{\mathrm{ex}}$ are defined by (131).

The above system is analogous to the well known in quantum mechanics Ehrenfest Theorem, [38, Sects. 7, 23]. Notice that the system of the equations of motion (92) departs from the corresponding system for point charges by having the averaged Lorentz density force instead of the Lorentz force at the exact position $\mathbf{r}^{\ell}(t)$.

Let us suppose now that for every $\ell$-th charge density $\left|\psi^{\ell}\right|^{2}$ and the corresponding current density $\mathbf{J}^{\ell}$ are localized in $a^{\ell}$-vicinity of the position $\mathbf{r}^{\ell}(t)$, and that the every localization scale $a^{\ell}$ is small compared with the typical variation scale $R_{\mathrm{EM}}$ of the EM field. Then if $R_{\mathrm{EM}}$ is bounded and $a^{\ell} \rightarrow 0$ we get

$$
\begin{gathered}
\left|\psi^{\ell}\right|^{2}(t, \mathbf{x}) \rightarrow \delta\left(\mathbf{x}-\mathbf{r}^{\ell}(t)\right), \\
\mathbf{v}^{\ell}(t, x)=\mathbf{J}^{\ell} / q^{\ell} \rightarrow \mathbf{v}^{\ell}(t) \delta\left(\mathbf{x}-\mathbf{r}^{\ell}(t)\right),
\end{gathered}
$$

where the coefficients before the Dirac delta-functions are determined by the charge normalization conditions (80) and relations (88). Using potential representations (81) we infer from (93) the convergence of the potentials $\varphi^{\ell}$ to the corresponding Coulomb's potentials, namely

$$
\begin{gathered}
\varphi^{\ell}(t, \mathbf{x}) \rightarrow \varphi_{0}^{\ell}(t, \mathbf{x})=\frac{q^{\ell}}{\left|\mathbf{x}-\mathbf{r}^{\ell}\right|}, \\
\nabla_{\mathbf{r}} \varphi^{\ell}(t, \mathbf{x}) \rightarrow \frac{q^{\ell}\left(\mathbf{x}-\mathbf{r}^{\ell}\right)}{\left|\mathbf{x}-\mathbf{r}^{\ell}\right|^{3}} \quad \text { as } a^{\ell} \rightarrow 0 .
\end{gathered}
$$

Hence, we can recast the equations of motion (92) as the system

$$
m^{\ell} \frac{\mathrm{d}^{2} \mathbf{r}^{\ell}}{\mathrm{d} t^{2}}=\mathrm{f}^{\ell}+\epsilon_{\mathrm{p} \ell}, \quad \ell=1, \ldots, N,
$$

where

$$
\mathrm{f}^{\ell}=\sum_{\ell^{\prime} \neq \ell} q^{\ell} \mathbf{E}_{0}^{\ell^{\prime}}+q^{\ell} \mathbf{E}_{\mathrm{ex}}\left(\mathbf{r}^{\ell}\right)+\frac{1}{\mathrm{c}} \mathrm{v}^{\ell} \times \mathbf{B}_{\mathrm{ex}}\left(\mathbf{r}^{\ell}\right)
$$


with small discrepancies $\epsilon_{\mathrm{p} \ell} \rightarrow 0$ as $a^{\ell} / R_{\mathrm{EM}} \rightarrow 0$. Notice that terms $\mathrm{f}^{\ell}$ in (95) coincide with the Lorentz forces. Since $\epsilon_{\mathrm{p} \ell} \rightarrow 0$ in the limit $a^{\ell} / R_{\mathrm{EM}} \rightarrow 0$ we see that the equations of motion (95) take approximately the form of Newton equations for point charges interacting via the Coulomb potential and corresponding Lorentz forces, namely for $\ell=1, \ldots, N$

$$
m^{\ell} \frac{\mathrm{d}^{2} \mathbf{r}^{\ell}}{\mathrm{d} t^{2}}=-\sum_{\ell^{\prime} \neq \ell} \frac{q^{\ell} q^{\ell^{\prime}}\left(\mathbf{r}^{\ell^{\prime}}-\mathbf{r}^{\ell}\right)}{\left|\mathbf{r}^{\ell^{\prime}}-\mathbf{r}^{\ell}\right|^{3}}+q^{\ell} \mathbf{E}_{\mathrm{ex}}\left(\mathbf{r}^{\ell}\right)+\frac{1}{\mathrm{c}} \mathrm{v}^{\ell} \times \mathbf{B}_{\mathrm{ex}}\left(\mathbf{r}^{\ell}\right) .
$$

To quantify the conditions of the remote interaction we make use of the explicit dependence on the size parameter $a$ of the nonlinearity $G^{\ell}=G_{a}^{\ell}$ as in (52) and take the size parameter as a spatial scale characterizing sizes of the fields $\psi_{a}^{\ell}$ and $\varphi_{a}^{\ell}$. The charges separation is measured roughly by a minimal distance $R_{\min }$ between any two charge positions. Another relevant spatial scale $R_{\text {ex }}$ measures a typical scale of spatial inhomogeneity of external fields near charges. Consequently, conditions of remote interaction are measured by the dimensionless ratio $a / R_{\mathrm{EM}}$ where the characteristic spatial scale $R_{\mathrm{EM}}=\min \left(R_{\min }, R_{\mathrm{ex}}\right)$ with the condition $a / R_{\mathrm{EM}} \ll 1$ to define the regime of remote interaction. To summarize, we observe that the exact equations of motion (92) form a basis for relating the field and point mechanics under an assumption that charge fields remain localized during time interval of interest. Notice the equations of motion (92) do not involve the nonlinear interaction terms $G^{\ell}$ in an explicit way justifying their characterization as "stealthy" in the regime of remote interactions. As to the assumption that the charge fields remain localized, it has to be verified based on the field equations (77) where the nonlinear interaction terms $G^{\ell}$ provide for cohesive forces for individual charges. The fact that they can do just that is demonstrated for a single charge represented as wave-corpuscle (19) as it accelerates in an external EM field. The last point naturally brings us to the second way of correspondence between the charges as fields and points when they interact, which we consider below.

\subsubsection{Many Interacting Wave-Corpuscles}

We show in this section that when charges are well separated their wave functions can be represented by wave-corpuscle formulas (19)-(96) which satisfy the field equations (77) with small discrepancies. More detailed presentation of that idea is as follows. Consider for simplicity a case when $\mathbf{A}_{\mathrm{ex}}=0$. As the first step we solve the Newton's equations of motion (96) and find the position functions $\mathbf{r}_{0}^{\ell}(t), \ell=1, \ldots, N$, which satisfy the following system of equations

$$
\begin{aligned}
m^{\ell} \frac{\mathrm{d}^{2} \mathbf{r}_{0}^{\ell}}{\mathrm{d} t^{2}} & =-q^{\ell} \nabla \varphi_{\mathrm{ex}, 0}^{\ell}\left(\mathbf{r}_{0}^{\ell}\right), \\
\mathbf{r}_{0}^{\ell}(0) & =\dot{\mathbf{r}}_{0}^{\ell}, \quad \frac{\mathrm{d} \mathbf{r}_{0}^{\ell}}{\mathrm{d} t}(0)=\dot{\mathbf{v}}_{0}^{\ell},
\end{aligned}
$$

where

$$
\varphi_{\mathrm{ex}, 0}^{\ell}(t, \mathbf{x})=\varphi_{\mathrm{ex}}(t, \mathbf{x})+\sum_{\ell^{\prime} \neq \ell} \frac{q^{\ell^{\prime}}}{\left|\mathbf{x}-\mathbf{r}^{\ell^{\prime}}\right|} .
$$

Obviously it coincides with (18) where $\mathbf{E}_{\mathrm{ex}}(\mathbf{x})=-\nabla \varphi_{\mathrm{ex}, 0}^{\ell}(t, \mathbf{x})$. The $\ell$-th equation (77), (82) for $\ell$-th charge has the form of field equations (9), (10) of motion of a single charge in an external EM field. As the second step we introduce the following wave-corpuscle 
representation similar to (19), (20):

$$
\begin{aligned}
\psi_{a}^{\ell}(t, \mathbf{x}) & =\mathrm{e}^{\mathrm{i} S^{\ell} / \chi} \dot{\psi}_{a}^{\ell}\left(\left|\mathbf{x}-\mathbf{r}_{0}^{\ell}\right|\right), \\
\varphi_{a}^{\ell}(t, \mathbf{x}) & =\dot{\varphi}_{a}^{\ell}\left(\mathbf{x}-\mathbf{r}_{0}^{\ell}\right),
\end{aligned}
$$

where the dependence on the size $a$ is given by (42) and $S^{\ell}(t, \mathbf{x})$ is defined in the terms of the potential $\varphi_{\mathrm{ex}, 0}^{\ell}$ as follows:

$$
\begin{aligned}
S^{\ell}(t, \mathbf{x}) & =\mathbf{p}_{0}^{\ell} \cdot\left(\mathbf{x}-\mathbf{r}_{0}^{\ell}\right)-\int_{0}^{t} \frac{\mathbf{p}_{0}^{\ell 2}}{2 m^{\ell}} \mathrm{d} t^{\prime}+q^{\ell} \int_{0}^{t} \varphi_{\mathrm{ex}, 0}^{\ell}\left(t^{\prime}, \mathbf{r}_{0}^{\ell}\right) \mathrm{d} t^{\prime}, \\
\mathbf{p}_{0}^{\ell} & =m^{\ell} \frac{\mathrm{d} \mathbf{r}_{0}^{\ell}}{\mathrm{d} t} .
\end{aligned}
$$

We assume the form factor $\dot{\psi}_{a}^{\ell}$ to decay exponentially, and, hence, its contributions for $\left|\mathbf{x}-\mathbf{r}_{0}^{\ell}\right| \gg a$ are negligible. Now we use the fact that the wave-corpuscle (19)-(20) is an exact solution to the fields equations (9), (10) if the EM potentials are linear functions of spatial variables. Since evidently the wave-corpuscle of the form (99) is localized about its center $\mathbf{r}^{\ell}(t)$ with the localization distance $a$ it provides an accurate approximation to the field equations in a vicinity of $\mathbf{r}^{\ell}(t)$ where the potentials of the external EM field and the Coulomb potential of other charges can be linearized. The Coulomb potentials can be linearized if the charges are well separated. Let us measure the charges separation roughly by the minimal distance $R_{\min }$ between any two charges in the system. Another relevant spatial scale $R_{\varphi}$ is a typical scale of spatial inhomogeneity of external fields around the charges. Hence, the linearization of the potentials around $\mathbf{r}^{\ell}(t)$ results an error of order $a^{2} / R^{2}$ where $R=\min \left(R_{\varphi}, R_{\min }\right)$. If the trajectories of the point charge do not collide and $R_{\min } \gg a$ we can define for every $\ell$ the wave-corpuscle $\psi^{\ell}(t, \mathbf{x})$ by formulas (99)-(100). Such a wavecorpuscle $\psi^{\ell}(t, \mathbf{x})$ is an exact solution to the auxiliary field equation with the field potential $\tilde{\varphi}_{\mathrm{ex}}^{\ell}$ obtained by the linearization of $\varphi_{\mathrm{ex}, 0}^{\ell}(t, \mathbf{x})$ at $\mathbf{r}^{\ell}(t)$. Since $\psi^{\ell}(t, \mathbf{x})$ is an exact solution to the auxiliary field equation, and it is localized about $\mathbf{r}^{\ell}(t)$ with the localization distance $a$ it produces a discrepancy of order $a^{2} / R^{2}$ in the original field equations. Note that the wave-corpuscle approximation (99) is based on trajectories $\mathbf{r}_{0}^{\ell}$ for the wave-corpuscle centers determined from (97) which involve the exact Coulomb potentials $\dot{\varphi}_{0}^{\ell}$ corresponding to the size parameter $a=0$, at the same time it involves the form factors and the potentials with $a>0$. Interestingly, an additional analysis of the exact motion equations (92) (where $\mathbf{B}_{\mathrm{ex}}=0$ ) shows that though the integral discrepancy decays as $a^{2} / R_{\varphi}^{2}$, the positions $\mathbf{r}^{\ell}(t)$ given by (88) are approximated by $\mathbf{r}_{0}^{\ell}(t)$ with accuracy of the order $a^{3} / R_{\varphi}^{3}$.

The above argument shows that the point charge mechanics features are transparently integrated into the fields $\left\{\psi_{a}^{\ell}, \varphi_{a}^{\ell}\right\}$ in (99), (100) via the de Broglie factor phases $S^{\ell}$ and the spatial shifts $\mathbf{r}_{0}^{\ell}$. Comparing the many charge system with a single charge in external field we observe that for the charge system the acceleration of the $\ell$-th charge center $\mathbf{r}_{0}^{\ell}(t)$ is determined not only by the Lorentz force due to the external field but also by electric interactions with the remaining charges according to the Coulomb's law.

We would like to point out that when analyzing the system of charges in the regime of remote interaction we do not use any specific form of the nonlinearities that keep charges wave functions spatially localized during the evolution. Note also that solutions of field equations (77) depend on the size parameter $a$ which is proportional to the radius of the wave-corpuscle and which enters the theory through the nonlinearity $G_{a}^{\ell}$, but the integral equations (92) do not involve explicit dependence on $a$. Equation (99) which describes the 
structure of the wave-corpuscle involves $a$ only through radial shape factors $\dot{\psi}^{\ell}=\dot{\psi}_{a}^{\ell}$ and through the electric potential $\dot{\varphi}^{\ell}=\dot{\varphi}_{a}^{\ell}$. The dependence of $\dot{\psi}_{a}^{\ell}$ on $a$ is explicitly singular at zero, as should be expected since in the singular limit $a \rightarrow 0$ the wave-corpuscle should turn into the point charge with the square of amplitude described by a delta function as in (3). Nevertheless, for arbitrary small $a>0$ the wave-corpuscle structure of every charge is preserved including its principal wave-vector. The dependence of $\dot{\varphi}_{a}^{\ell}$ on small $a$ can be described as a regular convergence to the classical singular Coulomb's potential, see (74) for details. That allows us to apply representation (99) to charges with small $a$ without compromising the accuracy of the description and, in fact, increasing the accuracy as $a \rightarrow 0$. Note that to obtain point charges equations of motion (95) it is sufficient to assume localization only for $\psi^{\ell}$.

\subsection{Symmetries and Conservation Laws}

In this section we discuss symmetries of the nonrelativistic Lagrangian, conservation laws which follow from the symmetries and the structure of the energy-momentum tensor.

\subsubsection{Free Single Charge}

The nonrelativistic Lagrangian $\hat{L}_{0}$ for a single charge is given by (7) and for a free charge we set $\mathbf{A}_{\mathrm{ex}}=0, \varphi_{\mathrm{ex}}=0$. Consequently, $\tilde{\nabla}=\nabla$ and the Euler-Lagrange field equations are given by (9), (10) with $\mathbf{A}_{\mathrm{ex}}=0, \varphi_{\mathrm{ex}}=0$. The Lagrangian $\hat{L}_{0}$ is a gauge invariant with respect to two kinds of gauge transformation: global and local, namely

$$
\psi \rightarrow \mathrm{e}^{\mathrm{i} \gamma} \psi
$$

where $\gamma$ is any real constant, and with respect to a reduced version of the second type gauge transformation

$$
\psi \rightarrow \mathrm{e}^{-\frac{\mathrm{i} q \lambda(t)}{x}} \psi, \quad \varphi \rightarrow \varphi+\partial_{t} \lambda(t)
$$

where the function $\lambda(t)$ may depend only on time.

To carry out a systematic analysis of conservation laws associated with the Lagrangian $\hat{L}_{0}$ defined by (7) via the Noether theorem, we need to find a Lie group of transformations which preserve it. The Lagrangian $\hat{L}_{0}$ is not invariant with respect to either the Lorentz or the Galilean groups of transformations. But a straightforward examination shows that $\hat{L}_{0}$ is invariant with respect to the following Galilean-gauge group of transformations

$$
\begin{aligned}
t^{\prime} & =t, \quad \mathbf{x}^{\prime}=\mathbf{x}-\mathbf{v} t, \\
\psi(t, \mathbf{x}) & =\mathrm{e}^{\mathrm{i} \frac{m}{2 \chi}\left(\mathbf{v}^{2} t^{\prime}+2 \mathbf{v} \cdot \mathbf{x}^{\prime}\right)} \psi^{\prime}\left(t^{\prime}, \mathbf{x}^{\prime}\right),
\end{aligned}
$$

with $\varphi(t, \mathbf{x})=\varphi^{\prime}\left(t^{\prime}, \mathbf{x}^{\prime}\right)$. One can also verify that the above transformations form an Abelian (commutative) group of transformations parametrized by the velocity parameter $\mathbf{v}$. It is curious to observe that according to the Galilean-gauge transformations (103), (104) the charge wave function does not transform as a scalar as in the relativistic case. These transformations are known, [18, Sect. 7.3], and were used, in particular, in studies of nonlinear Schrödinger equations, [42, Sect. 2.3].

The above defined Galilean-gauge group is naturally extended to the general inhomogeneous Galilean-gauge group by adding to it the group of spacial rotations $O$ and space-time 
translations $a^{\mu}$, namely

$$
\begin{aligned}
& t^{\prime}=t+\tau, \quad \mathbf{x}^{\prime}=O \mathbf{x}-\mathbf{v} t+\mathbf{a}, \\
& \psi^{\prime}\left(t^{\prime}, \mathbf{x}^{\prime}\right)=\mathrm{e}^{\mathrm{i} \frac{m}{2 \chi}\left[\mathbf{v}^{2}(t+\tau)-2 \mathbf{v} \cdot(O \mathbf{x}+\mathbf{a})\right]} \psi(t, \mathbf{x}), \\
& \varphi^{\prime}\left(t^{\prime}, \mathbf{x}^{\prime}\right)=\varphi(t, \mathbf{x}) .
\end{aligned}
$$

Carrying out the Noether currents analysis for the Lagrangian $\hat{L}_{0}$ we obtain 10 conservation laws which, as it turns out, can be formulated in terms of the canonical energy-momentum tensor $\stackrel{\circ}{\mathcal{T}}^{\mu \nu}$, which, in turn, is given by the following formula:

$$
\stackrel{\circ}{\mathcal{T}}^{\mu \nu}=\frac{\partial \hat{L}_{0}}{\partial \psi_{, \mu}} \psi_{, \nu}+\frac{\partial \hat{L}_{0}}{\partial \psi_{, \mu}^{*}} \psi_{, \nu}^{*}+\frac{\partial \hat{L}_{0}}{\partial \varphi_{, \mu}} \varphi_{, \nu}-\hat{L}_{0} g^{\mu \nu} .
$$

Namely, we get the total of ten conservation laws:

$$
\begin{aligned}
\partial_{\mu} \stackrel{\circ}{\mathcal{T}}^{\mu \nu} & =0 \text { - energy-momentum conserv., } \\
\mathcal{T}^{i j} & =\stackrel{\circ}{\mathcal{T}}^{j i}, \quad i, j=1,2,3 \text { - space angular momentum conserv., } \\
P^{i} & =\stackrel{\circ}{\mathcal{T}}^{0 i}=\frac{m}{q} J^{i}, \quad i=1,2,3 \text { - time-space angular momentum conserv. }
\end{aligned}
$$

The first four standard conservation laws (108) are associated with the Noether's currents with respect to space-time translations $a^{\mu}$. The second three conservation laws in (109) are associated with space rotation parameters $\xi$, and they turn into the symmetry of the energy-momentum tensor $\dot{\mathcal{T}}^{\mu v}$ for the spatial indices. The form of the last three conservation laws (110) is special to the nonrelativistic Lagrangian $\hat{L}_{0}$, and it is due to the Galileangauge invariance (104), (106). These relations indicate that the total momentum density $P^{i}$ is identically equal up to the factor $\frac{m}{q}$ to the microcurrent density $J^{i}$. This important identity is analogous to the kinematic representation $\mathbf{p}=m \mathbf{v}$ of the momentum $\mathbf{p}$ of a point charge. The proportionality of the momentum and the current is known to occur for systems governed by the nonlinear Schrödinger equations, [42, Sect. 2.3].

If charge is at rest, we find the following expressions for the total energy density of the dressed charge:

$$
\stackrel{\circ}{E}=\frac{\chi^{2}}{2 m}\left[(\nabla \dot{\psi})^{2}+G\left(\stackrel{\circ}{\psi}^{2}\right)\right]+\stackrel{\circ}{\psi}^{2} \stackrel{\circ}{ }-\frac{(\nabla \stackrel{\circ}{\varphi})^{2}}{8 \pi} .
$$

\subsubsection{Many Interacting Charges}

We discuss now briefly the structure of the Lagrangian and the energy-momentum tensor. The Lagrangian $\hat{\mathcal{L}}$ defined by (75), (76) is gauge invariant with respect to (101) and the second gauge transformations,

$$
\psi \rightarrow \mathrm{e}^{-\frac{\mathrm{i} e \lambda(x)}{\chi \mathrm{c}}} \psi, \quad A^{\mu} \rightarrow A^{\mu}+\partial^{\mu} \lambda .
$$

In this case every 4-current $J^{\ell \mu}=\left(c \rho^{\ell}, \mathbf{J}^{\ell}\right)$ defined by relations (78) satisfies the conservation/continuity equations (79) and the charge normalization condition (80).

The presence of the external EM field turns the dressed charge into an open system with consequent subtleties in the treatment of the energy-momentum, [6]. An instrumental element in the analysis of the energy-momentum tensor is its partition between the charge and 
the EM field. In carrying out such a partition we are guided by two principles: (i) both the energy-momenta tensors and the forces have to be gauge invariant; (ii) the forces must be of the Lorentz form. The second principle is evidently special to the EM system consisting of the charge and the EM field. In the regime of remote interactions it makes sense to introduce dressed charges and attribute to every charge its EM field via the potential $\varphi^{\ell}$ as defined by relations (77) and (81). Based on the potentials $\varphi^{\ell}$ we define the corresponding energy-momentum tensor $\Theta^{\ell \mu \nu}$. The energy-momentum tensor can be written as a sum of gauge invariant energy-momentum tensors $\tilde{T}^{\mu v}$ and $\Theta^{\mu v}$ for the charge and the EM field respectively. The following conservations laws for the individual charges and the EM field hold:

$$
\begin{aligned}
& \partial_{\mu} \tilde{T}^{\ell \mu \nu}=f^{\ell v}+f_{\mathrm{ex}}^{\ell v}, \\
& \partial_{\mu} \Theta^{\ell \mu \nu}=-f^{\ell v},
\end{aligned}
$$

where

$$
\begin{aligned}
& f^{\ell v}=\frac{1}{\mathrm{c}} J_{\mu}^{\ell} F^{\ell \nu \mu}=\left(\frac{1}{\mathrm{c}} \mathbf{J}^{\ell} \cdot \mathbf{E}, \rho^{\ell} \mathbf{E}\right), \\
& f_{\mathrm{ex}}^{\ell v}=\left(\frac{1}{\mathrm{c}} \mathbf{J}^{\ell} \cdot \mathbf{E}_{\mathrm{ex}}, \rho^{\ell} \mathbf{E}_{\mathrm{ex}}+\frac{1}{\mathrm{c}} \mathbf{J}^{\ell} \times \mathbf{B}_{\mathrm{ex}}\right) .
\end{aligned}
$$

We would like to point out that the energy-momentum conservation equations (113)-(115) can be viewed as equations of motion in an elastic continuum, [29, Sect. 6.4, (6.56), (6.57)], similar to the case of kinetic energy-momentum tensor for a single relativistic particle, [33, Sect. 37, (3.24)]. It is important to remember though that in contrast to the point mechanics the equations of motion (113)-(115) must always be complemented with the field equations (77) without which they do not have to hold and are not alone sufficient to determine the motion. We also recognize in $f^{\ell v}$ and $f_{\mathrm{ex}}^{\ell v}$ in the equations of motion (113)-(115) respectively the Lorentz force densities for the charge in the EM field of charges and the same for the external EM field. Observe that (113)-(114) satisfy manifestly the Newton's principle "action equals reaction" for all involved densities at every point of the space-time. Namely, the $\ell$-the charge EM field $F^{\ell v \mu}$ acts upon the $\ell$-the charge with the force density $f^{\ell v}$ according to (113) whereas the action of the same charge upon its EM field $F^{\ell v \mu}$ is exactly opposite $-f^{\ell v}$ in (114).

\subsection{Stability Issues}

A comprehensive analysis of the stability is complex, involved and beyond the scope of this paper. Nevertheless, we would like to give a concise consideration to three aspects of stability for well separated charges in the nonrelativistic regime: (i) no "blow-up" or "collapse"; (ii) preservation with high accuracy of the form of a wave-corpuscle solution for a limited time; (iii) preservation of spatial localization for certain solutions on long time intervals.

Here is an argument for why there can not be a "blow-up" in finite time. A "blow-up" is an issue since the nonlinearity $G^{\prime}(s)$ provides focusing properties with consequent solitonlike solutions $\dot{\psi}^{\ell}, \stackrel{\varphi}{\varphi}^{\ell}$. In our model the possibility of "blow up" is excluded when we define $\left[G_{a}^{\ell}\right]^{\prime}$ to be a constant for large amplitudes of the fields, namely for $s \geq\left(\dot{\psi}^{\ell}\right)^{2}(0)$ as in (47). This factor combined with the charge normalization condition (11) implies that the energy 
is bounded from below. Indeed, the energy of a free charge can be written in the form

$$
\mathcal{E}(\psi, \varphi)=\int_{\mathbb{R}^{3}} \frac{|\nabla \varphi|^{2}}{8 \pi}+\frac{\chi^{2}}{2 m}\left[|\nabla \psi|^{2}+G\left(|\psi|^{2}\right)\right] \mathrm{d} \mathbf{x},
$$

where $\varphi=\varphi_{\psi}$ is determined from (81). In view of relations (47) the nonlinearity derivative $G^{\prime}(s)$ is bounded, implying $G\left(|\psi|^{2}\right) \geq-C|\psi|^{2}$ for a constant $C$. That combined with the charge normalization condition (11) implies boundedness of the energy from below, namely

$$
\mathcal{E}(\psi, \varphi) \geq-C \quad \text { for all } \psi, \varphi,\|\psi\|^{2}=\int_{\mathbb{R}^{3}}|\psi|^{2} \mathrm{~d} \mathbf{x}=1 .
$$

A similar argument in the case of many interacting charges also shows that the energy is bounded from below. Since energy is a conserved quantity, using the boundedness of the energy from below one can prove along lines of [24] the global existence of a unique solution $\psi^{\ell}(t, \mathbf{x}), \varphi^{\ell}(t, \mathbf{x})$ to $(77),(82)$ for all times $0 \leq t<\infty$ for given initial data $\psi^{\ell}(0, \mathbf{x})$. Note that finite time stability does not require charges center to be separated, they may overlap as in hydrogen atom model. This may lead to a substantial change of size and shape, for example in hydrogen atom electron size shrinks several times thanks to attraction to proton.

The second aspect of the stability is about a preservation of the wave-corpuscle shape with high accuracy for limited times. The discrepancies in the field equations (22) after substitution of wave-corpuscles are of the order $|q \| \bar{\varphi}| a^{2} / R^{2}$ for the charge in an external EM field, where $|\bar{\varphi}|$ is a measure of variation of the external potential $\varphi_{\mathrm{ex}}$ around trajectory. Therefore the fields $\psi, \varphi$ have to be close to the wave-corpuscle of the form (19) on time intervals of order $|q \| \bar{\varphi}| a^{2} /\left(\chi R^{2}\right)$ where $R$ is a spatial scale of inhomogeneity of the external field, and $|q||\bar{\varphi}|$ is a global variation of the external field potential energy near the trajectory of the wave-corpuscle.

The third aspect is a stability on very long time intervals which is understood in a broader sense, namely when a charge maintains its spatial localization without necessarily preserving the exact form of a wave-corpuscle. It is shown in Sect. 2.6 that such a broad localization assumption is sufficient to identify the corresponding point charge trajectory. Now let us consider the following argument for the charge stability based on properties of the energy. For simplicity let us consider a single free charge described by (9)-(10) with zero external fields with energy (116). The energy conservation law implies

$$
\mathcal{E}(\psi(t), \varphi(t))=\mathcal{E}(\psi(0), \varphi(0)), \quad \text { for all } 0 \leq t<\infty .
$$

Note that the rest solution $\stackrel{\wp}{\psi}$ as in (12), (13) is a critical point of $\mathcal{E}$ defined by (116). Let us assume that the rest solution $\stackrel{i}{\mathrm{i}}$ is the global minimum under the charge normalization constraint, namely

$$
\mathcal{E}\left(\dot{\psi}, \varphi_{\psi}\right)=\min _{\|\psi\|=1} \mathcal{E}\left(\psi, \varphi_{\psi}\right)=\mathrm{E}_{0} .
$$

Consider then the initial data $\psi_{0}$ for (9)-(10) at $t=0$ that (i) satisfies the charge normalization condition (11); (ii) is close to $\dot{\psi}$ and has almost the same energy, i.e. $\left|\mathcal{E}(\psi(0), \varphi(0))-\mathrm{E}_{0}\right| \ll 1$. Note that since every spatial shift $\dot{\psi}(\mathbf{x}-\mathbf{r}), \varphi_{\dot{\psi}}(\mathbf{x}-\mathbf{r})$ of $\dot{\psi}(\mathbf{x})$, $\varphi_{\dot{\psi}}(\mathbf{x})$ produces fields satisfying the charge normalization condition (11) and of the same energy, the minimum in (119) has to be degenerate. But if we assume that all the degeneracy is due to the spatial translations, rotations and the multiplication by $\mathrm{e}^{i s}$, then the condition $\left|\mathcal{E}(\psi(t), \varphi(t))-\mathrm{E}_{0}\right| \ll 1$ though allowing for spatial translation of $\dot{\psi}(\mathbf{x}), \varphi_{\dot{\psi}}(\mathbf{x})$ to large distances and times, still implies that form of $|\psi(t, \mathbf{x}-\mathbf{r}(t))|, \varphi(t, \mathbf{x}-\mathbf{r}(t))$ has to be almost 
the same as the form of $\dot{\psi}(\mathbf{x}), \stackrel{\varphi}{\varphi}(\mathbf{x})$. The same argument works for a local minimum that is non-degenerate modulo spatial translations, rotations and the multiplication by $\mathrm{e}^{\mathrm{i} s}$.

\section{Relativistic Theory}

In this section we give a sketch of the relativistic theory for a single free charge and many interacting charges with more detailed exposition available in [6].

\subsection{Single Free Relativistic Charge}

We describe a bare single elementary charge by a complex-valued scalar field $\psi=\psi(x)=$ $\psi(t, \mathbf{x})$, where $x=(t, \mathbf{x}) \in \mathbb{R}^{4}$ is the space-time variable and it is coupled with the EM field described by its 4-potential $A^{\mu}=(\varphi, \mathbf{A})$. The relativistic Lagrangian of a single charge is

$$
\begin{aligned}
L_{0}\left(\psi, A^{\mu}\right)= & \frac{\chi^{2}}{2 m}\left\{\frac{1}{\mathrm{c}^{2}}\left|\tilde{\partial}_{t} \psi\right|^{2}-|\tilde{\nabla} \psi|^{2}-\kappa_{0}^{2}|\psi|^{2}-G\left(\psi^{*} \psi\right)\right\} \\
& +\frac{1}{8 \pi}\left[\left(\nabla \varphi+\frac{1}{\mathrm{c}} \partial_{t} \mathbf{A}\right)^{2}-(\nabla \times \mathbf{A})^{2}\right],
\end{aligned}
$$

where the covariant derivatives $\tilde{\partial}_{t}$ and $\tilde{\nabla}$ are defined by the formulas

$$
\psi_{; \mu}=\left(\frac{1}{\mathrm{c}} \tilde{\partial}_{t} \psi,-\tilde{\nabla} \psi\right), \quad \tilde{\partial}_{t}=\partial_{t}+\frac{\mathrm{i} q \varphi}{\chi}, \tilde{\nabla}=\nabla-\frac{\mathrm{i} q^{\ell} \mathbf{A}}{\chi \mathrm{c}}
$$

and

$$
\kappa_{0}=\frac{m \mathrm{c}}{\chi}
$$

The Lagrangian $\mathcal{L}$ defined by (120) is manifestly local, Lorentz invariant, and gauge invariant with respect to the second-kind (local) gauge transformation

$$
\psi \rightarrow \mathrm{e}^{-\frac{\mathrm{i} q \lambda(x)}{\chi \mathrm{c}}} \psi, \quad A^{\mu} \rightarrow A^{\mu}+\partial^{\mu} \lambda(x),
$$

as well as with respect to the group of global (the first-kind) gauge transformations

$$
\psi \rightarrow \mathrm{e}^{-\mathrm{i} q \lambda} \psi, \quad A^{\mu} \rightarrow A^{\mu},
$$

for any real numbers $\lambda$. Using the gauge invariance of the Lagrangian $L_{0}$ we derive the following expression for the conserved charge 4-vector micro-current $J^{\mu}=(\rho \mathrm{c}, \mathbf{J})$

$$
\begin{aligned}
\rho & =-\frac{q|\psi|^{2}}{m \mathrm{c}^{2}}\left(\chi \operatorname{Im} \frac{\partial_{t} \psi}{\psi}+q \varphi\right), \\
\mathbf{J}^{\ell} & =\frac{q|\psi|^{2}}{m}\left(\chi \operatorname{Im} \frac{\nabla \psi}{\psi}-\frac{q \mathbf{A}}{\mathrm{c}}\right),
\end{aligned}
$$

satisfying the conservation/continuity equations $\partial_{\nu} J^{v}=0$ or

$$
\partial_{t} \rho+\nabla \cdot \mathbf{J}=0 .
$$


The formulas for the 4-microcurrent density $J^{\mu}=(\rho \mathbf{c}, \mathbf{J})$ are well known in the literature, see for instance, [46, (11.3)], [30, Sect. 3.3, (3.3.27), (3.3.34), (3.3.35)]. As a consequence of the continuity equations (155) the space integral of every $\rho(x)$ is a conserved quantity, which we assign to be exactly $q$, i.e. we assume the following charge normalization

$$
\int_{\mathbb{R}^{3}} \frac{\rho(x)}{q} \mathrm{~d} \mathbf{x}=-\frac{1}{m \mathrm{c}^{2}} \int_{\mathbb{R}^{3}}\left(\chi \operatorname{Im} \frac{\partial_{t} \psi}{\psi}+q \varphi\right)|\psi|^{2} \mathrm{~d} \mathbf{x}=1 .
$$

To emphasize the fact that our charge is always coupled with the EM field we name the pair $\left\{\psi, A^{\mu}\right\}$ dressed charge. So whenever we use the term dressed charge we mean the charge and the EM field as an inseparable entity.

The Euler-Lagrange equations for the Lagrangian $L_{0}$ are

$$
\begin{aligned}
& {\left[\mathrm{c}^{-2} \tilde{\partial}_{t}^{2}-\tilde{\nabla}^{2}+\kappa^{2}+G^{\prime}\left(|\psi|^{2}\right)\right] \psi=0,} \\
& \nabla \cdot\left(\frac{1}{\mathrm{c}} \partial_{t} \mathbf{A}+\nabla \varphi\right)=-4 \pi \sum_{\ell} \rho^{\ell}, \\
& \nabla \times(\nabla \times \mathbf{A})+\frac{1}{\mathrm{c}} \partial_{t}\left(\frac{1}{\mathrm{c}} \partial_{t} \mathbf{A}+\nabla \varphi\right)=\frac{4 \pi}{\mathrm{c}} \sum_{\ell} \mathbf{J}^{\ell} .
\end{aligned}
$$

with usual relations between EM potentials and fields

$$
\mathbf{E}=-\nabla \varphi-\frac{1}{\mathrm{c}} \partial_{t} \mathbf{A}, \quad \mathbf{B}=\nabla \times \mathbf{A} .
$$

Observe that (129)-(130), which are a detalization of (6), coincide exactly with the Maxwell equation written for the potentials.

Evidently the Lagrangian $L_{0}$ defined by the formulas (120) is obtained from the KleinGordon Lagrangian, [20, Sects. 7.1, 11.2], [7, Sect. III.3], by adding to it the nonlinear term $G\left(\psi^{*} \psi\right)$. The Lagrangian expression indicates that the charge is coupled to the EM field through the covariant derivatives, and such a coupling is well known and called minimal. The Klein-Gordon Lagrangian is a commonly used model for a relativistic spinless charge, and the introduced nonlinearity $G\left(\psi^{*} \psi\right)$ can provide for a binding self-force. Nonlinear alterations of the Klein-Gordon Lagrangian were considered in the literature, see, for instance, [20, Sects. 11.7, 11.8] and [8], for rigorous mathematical studies. Our way to choose of the nonlinearity $G\left(\psi^{*} \psi\right)$ differs from those.

Let us take a closer look at the components of the Lagrangian (120). It involves constants $\kappa_{0}, \mathrm{c}, \chi$ and $m$ and, acting similarly to the case of the Klein-Gordon equation for a relativistic particle (see [32, Sects. 1, 18, 19]), we introduce a fundamental frequency $\omega_{0}=\frac{m \mathrm{c}^{2}}{\chi}$ relating it to the above constants by formulas (154). A key component of the Lagrangian in (120) is a real-valued nonlinear function $G(s)$ providing for the charge cohesive self-interaction. The second part of the expression (120) is the standard Lagrangian of the EM field coupled to the charge via the covariant derivatives. Observe that the Lagrangian $L_{0}$ is manifestly (i) local; (ii) Lorentz and gauge invariant, and (iii) it has a local nonlinear term providing for a cohesive self-force similar to the Poincaré force for the Lorentz-Poincaré model of an extended charge.

Since a single charge is coupled at all times to the EM field we always deal with the system "charge-EM field" or, shortly, with the dressed charge. As in the nonrelativistic case 
dressed charge is considered to be at rest at the origin $\mathbf{x}=\mathbf{0}$ if it is a radial solution to the field equations (128)-(130) of the following special form

$$
\begin{aligned}
& \psi(t, \mathbf{x})=\mathrm{e}^{-\mathrm{i} \omega_{0} t} \stackrel{\circ}{\psi}(|\mathbf{x}|), \quad \varphi(t, \mathbf{x})=\stackrel{\circ}{\varphi}(|\mathbf{x}|), \\
& \mathbf{A}(t, \mathbf{x})=\mathbf{0}, \quad \omega_{0}=\frac{m \mathrm{c}^{2}}{\chi},
\end{aligned}
$$

and we refer to such a solution as $\omega_{0}$-static. Plugging the above expression into (128)-(130) we get the following system of equations

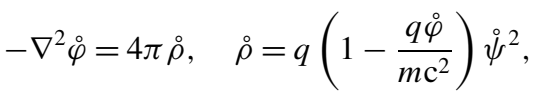

$$
\begin{aligned}
& -\nabla^{2} \dot{\psi}+\frac{m \dot{\varphi}}{\chi^{2}} q\left(2-\frac{q \dot{\varphi}}{m \mathrm{c}^{2}}\right) \dot{\psi}+G^{\prime}\left(|\dot{\psi}|^{2}\right) \dot{\psi}=0,
\end{aligned}
$$

where $\Delta=\nabla^{2}$ is the Laplace operator, this system is relativistic version of (12)-(13). We refer to the state of the dressed charge of the form (132) as $\omega_{0}$-static. Using Green's function to solve (133) we see that the charge form factor $\not$ determines the Coulomb-like potential $\stackrel{\circ}{\varphi}=\stackrel{\circ}{\dot{\psi}}_{\dot{\psi}}$ by the formula

$$
\begin{aligned}
& \stackrel{\circ}{\varphi} \stackrel{\circ}{\varphi}_{\dot{\psi}}=4 \pi q\left(-\nabla^{2}+\frac{4 \pi q^{2}}{m \mathrm{c}^{2}} \dot{\psi}^{2}\right)^{-1} \dot{\psi}^{2} . \\
& -\nabla^{2} \dot{\psi}+\frac{m \stackrel{\circ}{\dot{\psi}}^{2}}{\chi^{2}} q\left(2-\frac{q \stackrel{\circ}{\dot{\psi}}_{\dot{\psi}}}{m \mathrm{c}^{2}}\right) \dot{\psi}+G^{\prime}\left(|\dot{\psi}|^{2}\right) \stackrel{\circ}{\psi}=0 .
\end{aligned}
$$

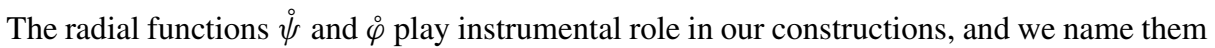
respectively charge form factor and form factor potential. As it follows from (133), the charge form factor $\dot{\psi}=\dot{\varphi}_{\dot{\psi}}$ determines the form factor potential $\stackrel{\circ}{\varphi}$ by the formula (135). Consequently, plugging in the above expression into (134) we get the nonlinear equation (136). Equation (136) signifies a complete balance (equilibrium) of the three forces acting upon the resting charge: (i) internal elastic deformation force associated with the term $-\Delta \dot{\psi}$; (ii) charge's electromagnetic self-interaction force associated with the term $\frac{m \dot{\varphi}_{\dot{\psi}}}{\chi^{2}}\left(2 q-\frac{q^{2} \dot{\varphi}_{\dot{\psi}}}{m \mathrm{c}^{2}}\right) \dot{\psi}$; (iii) internal nonlinear self-interaction of the charge associated with the term $G^{\prime}\left(|\dot{\psi}|^{2}\right) \dot{\psi}$. We refer to (136) as charge equilibrium equation. Importantly, as in non-relativistic case, the charge equilibrium equation (136) establishes an explicit relation between the form factor $\stackrel{\circ}{\psi}$ and the self-interaction nonlinearity $G$.

Hence being given the form factor $\dot{\psi}$ we can find from the equilibrium equation (136) the self-interaction nonlinearity $G$ which exactly produces this factor under an assumption that $\dot{\psi}(r)$ is a nonnegative, monotonically decaying and sufficiently smooth function of $r \geq 0$. Having fixed the nonlinear self-interaction $G$ based on the charge equilibrium equation (136) the challenge is to figure out the dynamics of the charge as it interacts with other charges or is acted upon by an external EM field and hence accelerates. The nonlinear self-interaction $G$ evidently brings into the charge model non-electromagnetic forces, the necessity of which for a consistent relativistic electromagnetic theory was argued convincingly by W. Pauli in [33, Sect. 63]. 
For the fundamental pair $\{\dot{\psi}, \stackrel{\circ}{\varphi}\}$ the corresponding microcharge density is defined by (125) turns into

$$
\rho=\rho(|\mathbf{x}|)=q\left(1-\frac{q \stackrel{\circ}{(}(|\mathbf{x}|)}{m \mathrm{c}^{2}}\right) \dot{\psi}^{2}(|\mathbf{x}|) .
$$

The symmetric and gauge invariant energy-momenta tensors $T^{\mu v}$ and $\Theta^{\mu v}$ for, respectively, the charge and the EM field are as follows, [6],

$$
\begin{aligned}
& T^{\mu \nu}=\frac{\chi^{2}}{2 m}\left\{\left[\psi^{; \mu *} \psi^{; v}+\psi^{; \mu} \psi^{; \nu *}\right]-\left[\psi_{; \mu}^{*} \psi^{; \mu}-\kappa_{0}^{2} \psi^{*} \psi-G\left(\psi^{*} \psi\right)\right] g^{\mu \nu}\right\}, \\
& \Theta^{\mu \nu}=\frac{1}{4 \pi}\left(g^{\mu \gamma} F_{\gamma \xi} F^{\xi \nu}+\frac{1}{4} g^{\mu \nu} F_{\gamma \xi} F^{\gamma \xi}\right),
\end{aligned}
$$

where $g^{\mu \nu}$ is the Minkowski tensor, $F^{\gamma \xi}$ is EM tensor as in (114). The energy conservation equations are given by

$$
\partial_{\mu} T^{\mu v}=f^{v}, \quad \partial_{\mu} \Theta^{\mu v}=-f^{v},
$$

where

$$
f^{v}=\frac{1}{\mathrm{c}} J_{\mu} F^{v \mu}=\left(\frac{1}{\mathrm{c}} \mathbf{J} \cdot \mathbf{E}, \rho \mathbf{E}+\frac{1}{\mathrm{c}} \mathbf{J} \times \mathbf{B}\right)
$$

is the Lorentz force density.

Using the Lorentz invariance of the system we can obtain, as it is often done, a representation for the dressed charge moving with a constant velocity $\mathbf{v}$ simply by applying to the rest solution (132) the Lorentz transformation from the original "rest frame" to the frame in which the "rest frame" moves with the constant velocity v. Namely, introducing

$$
\begin{aligned}
& \beta=\frac{\mathbf{v}}{\mathrm{c}}, \quad \beta=|\beta|, \quad \gamma=\left(1-\left(\frac{v}{\mathrm{c}}\right)^{2}\right)^{-1 / 2}, \\
& \omega=\gamma \omega_{0}, \quad \mathbf{k}=\gamma \beta \frac{\omega_{0}}{\mathrm{c}},
\end{aligned}
$$

we obtain the following representation for the dressed charge moving with velocity $\mathbf{v}$

$$
\begin{aligned}
& \psi(t, \mathbf{x})=\mathrm{e}^{-\mathrm{i}(\omega t-\mathbf{k} \cdot \mathbf{x})} \stackrel{\circ}{\psi}\left(\mathbf{x}^{\prime}\right), \\
& \varphi(t, \mathbf{x})=\gamma \stackrel{\circ}{ }\left(\left|\mathbf{x}^{\prime}\right|\right), \quad \mathbf{A}(t, \mathbf{x})=\gamma \beta \stackrel{\circ}{ }\left(\left|\mathbf{x}^{\prime}\right|\right),
\end{aligned}
$$

where

$$
\mathbf{x}^{\prime}=\mathbf{x}+\frac{\gamma-1}{\beta^{2}}(\beta \cdot \mathbf{x}) \beta-\gamma \mathbf{v} t, \quad \text { or } \quad \mathbf{x}_{\|}^{\prime}=\gamma\left(\mathbf{x}_{\|}-\mathbf{v} t\right), \quad \mathbf{x}_{\perp}^{\prime}=\mathbf{x}_{\perp},
$$

$\mathbf{x}_{\|}$and $\mathbf{x}_{\perp}$ refer, respectively, to the components of $\mathbf{x}$ parallel and perpendicular to the velocity $\mathbf{v}$, with the fields given by

$$
\begin{aligned}
& \mathbf{E}(t, \mathbf{x})=-\gamma \nabla \stackrel{\circ}{\varphi}\left(\left|\mathbf{x}^{\prime}\right|\right)+\frac{\gamma^{2}}{\gamma+1}\left(\beta \cdot \nabla \stackrel{\varphi}{\gamma}\left(\left|\mathbf{x}^{\prime}\right|\right)\right) \beta, \\
& \mathbf{B}(t, \mathbf{x})=\gamma \beta \times \nabla \stackrel{\circ}{ }\left(\left|\mathbf{x}^{\prime}\right|\right) .
\end{aligned}
$$


The above formulas provide for a solution to the field equations (128)-(130). They show, in particular, that the fields of the dressed charge contract by the factor $\gamma$ as it moves with the velocity $\mathbf{v}$ compared to their rest state. The second factor in the formula (144) for $\psi$ involves the form factor $\dot{\psi}(r)$, which is a monotonically decreasing function of $r \geq 0$ decaying at infinity. For such a form factor the form factor potential $\stackrel{\circ}{\varphi}(r)$ decays at infinity as the Coulomb's potential as it follows from (135), i.e. $\stackrel{\leftrightarrow}{\varphi}(r) \sim q r^{-1}$ for large $r$. Consequently, the dressed charge moving with constant velocity $\mathbf{v}$ as described by (144)-(145) remains well localized and does not disperse in the space at all times justifying its characterization as a wave-corpuscle. Observe that our relations (148) under the assumption that $\chi=\hbar$ are identical to those of a free charge as described by the Klein-Gordon equation, [32, Sects. 1, 18], but there are several significant differences between the two models which are as follows. First of all, our charge is a dressed charge described by the pair $\left\{\psi, A^{\mu}\right\}$. From the very outset it includes the EM field as its inseparable part whereas the Klein-Gordon model describes a free charge by a complex-valued wave function $\psi$ which is not coupled to its own EM field (not to be confused with an external EM field). Second, our free dressed charge when it moves, evidently preserves its shape up to the natural Lorentz construction whereas any wavepacket satisfying Klein-Gordon equation spreads out in the course of time.

The first oscillatory exponential factor in (144) is the de Broglie plane wave of the frequency $\omega=\omega(\mathbf{k})$ and the de Broglie wave-vector $\mathbf{k}$, satisfying

$$
\omega^{2}-\mathrm{c}^{2} \mathbf{k}^{2}=\omega_{0}^{2}, \quad \chi \omega_{0}=m \mathrm{c}^{2}
$$

Notice that the equalities (142)-(143) readily imply the following relations between $\omega, \mathbf{k}$ and $\mathbf{v}$

$$
\omega=\omega(\mathbf{k})=\sqrt{\omega_{0}^{2}+\mathrm{c}^{2} \mathbf{k}^{2}}, \quad \mathbf{v}=\nabla_{\mathbf{k}} \omega(\mathbf{k}),
$$

where $\omega_{0}=\omega$ is given by (154). Notice that the above relations show, in particular, that for the freely moving dressed charge defined by equalities (142)-(145) its velocity $\mathbf{v}$ equals exactly the group velocity $\nabla_{\mathbf{k}} \omega(\mathbf{k})$ computed for the de Broglie wave vector $\mathbf{k}$. This fact clearly points to the wave origin of the charge kinematics as it moves in the three dimensional space continuum with the dispersion relation $\omega=\sqrt{\omega_{0}^{2}+\mathrm{c}^{2} \mathbf{k}^{2}}$. Notice that this dispersion relation is identical to the dispersion relation of the Klein-Gordon equation as a model for a free charge, [32, Sect. 18]. Based on the Lagrangian $L_{0}$ we found the symmetric energy-momentum tensor, which shows that the dressed charge moving with a constant velocity $\mathbf{v}$ and described by (142)-(145) has momentum $P$ and energy $E$, which satisfy the Einstein-de Broglie relations

$$
\begin{aligned}
& \mathrm{P}=\hbar \mathbf{k}=\gamma \beta \frac{\hbar \omega_{0}}{\mathrm{c}}=\gamma \tilde{m} \mathbf{v} \\
& \mathrm{E}=\hbar \omega=\hbar \gamma \omega_{0}=\gamma \tilde{m} \mathrm{c}^{2}=\mathrm{c} \sqrt{\mathrm{P}^{2}+\tilde{m}^{2} \mathrm{c}^{2}} \\
& \mathrm{E}=\hbar \omega, \quad \mathrm{P}=\hbar \mathbf{k} .
\end{aligned}
$$

The total 4-momentum $P$ is obtained from its density by integration over the space $\mathbb{R}^{3}$, since the dressed charge is a closed system, its total 4-momentum $\mathrm{P}^{v}=(\mathrm{E}, \mathrm{cP})$ is 4-vector. We would like to point out that, though the above argument used to obtain the relations (151) is rather standard, in our case relations (151) are deduced rather than rationally imposed. 


\subsection{Many Interacting Charges}

Relativistic theory of many interacting point particles is known to have fundamental difficulties. "The invariant formulation of the motion of two or more interacting particles is complicated by the fact that each particle will have a different proper time. ... No exact general theory seems to be available", [7, Sect. II.1, System of colliding particles]. Some of these difficulties are analyzed by H. Goldstein in his classical monograph, [17, Sect. 7.10]: "The great stumbling block however is the treatment of the type of interaction that is so natural and common in nonrelativistic mechanics - direct interaction between particles. ... In special relativity, where signals cannot travel faster than the speed of light, action-at-a-distance seems outlawed. And in a certain sense this seems to be the correct picture. It has been proven that if we require certain properties of the system to behave in the normal way (such as conservation of total linear momentum), then there can be no covariant direct interaction between particles except through contact forces." Another argument, due to von Laue, [44], on the incompatibility of the relativity with any finite dimensional mechanical system was articulated by W. Pauli, [33, Sect. 45]: “... This in itself raised strong doubts as to the possibility of introducing the concept of a rigid body into relativistic mechanics ${ }^{247}$. The final clarification was brought about in a paper by Laue ${ }^{248}$, who showed by quite elementary arguments that the number of kinematic degrees of freedom of a body cannot be limited, according to the theory of relativity."

Now we ask ourselves what features of point charges mechanics can be integrated into a relativistic mechanics of fields? It seems that the above arguments by Goldstein, von Laue and Pauli completely rule out any Lagrangian mechanics with finitely many degrees of freedom even as an approximation because of its incompatibility with a basic relativity requirement for the signal speed not to exceed the speed of light. On the constructive side, these arguments suggest that (i) the EM field has to be an integral part of charges mechanics, (ii) every charge of the system has to be some kind of elastic continuum coupled to the EM field. We anticipate though that point mechanics features that can be integrated into a relativistic field mechanics are limited and have subtler manifestation compared to the nonrelativistic theory. We expect point mechanics features to manifest themselves in (i) identification of the energy-momentum tensor for every individual bare charge; (ii) certain partition of the EM field into a sum of EM fields attributed to individual charges with consequent formation of dressed charges, that is bare charges with attached to them EM fields.

In the theory proposed here we address the above challenges by (i) the principal departure from the concept of point charge, which is substituted by a concept of wave-corpuscle described by a complex valued function in the space-time; (ii) requirement for every charge to interact directly to only the EM field implying that different charges interact only via the EM field. We describe $\ell$-th elementary spinless charge by a complex-valued scalar field $\psi^{\ell}=\psi^{\ell}(t, \mathbf{x})$, where $x=(t, \mathbf{x}) \in \mathbb{R}^{4}$ is the space-time variable and the EM field by its 4-potential $A^{\mu}=(\varphi, \mathbf{A})$. The system Lagrangian $\mathcal{L}$ is a function of the 4-potential and the covariant derivatives $\psi_{; \mu}^{\ell}$ defined by the following formulas

$$
\begin{aligned}
& \psi_{; \mu}^{\ell}=\left(\frac{1}{\mathrm{c}} \tilde{\partial}_{t}^{\ell} \psi^{\ell},-\tilde{\nabla}^{\ell} \psi^{\ell}\right), \quad \text { where } \\
& \tilde{\partial}_{t}^{\ell}=\partial_{t}+\frac{\mathrm{i} q^{\ell} \varphi}{\chi}, \tilde{\nabla}^{\ell}=\nabla-\frac{\mathrm{i} q^{\ell} \mathbf{A}}{\chi \mathrm{c}},
\end{aligned}
$$


and $\psi^{\ell *}$ denotes complex conjugate to $\psi^{\ell}$, c is the speed of light. The system Lagrangian $\mathcal{L}$ has the following form:

$$
\begin{aligned}
\mathcal{L}\left(\left\{\psi^{\ell}, \psi_{; \mu}^{\ell}\right\}, A^{\mu}\right)= & \sum_{\ell=1}^{N} \frac{\chi^{2}}{2 m^{\ell}}\left\{\psi_{; \mu}^{\ell *} \psi^{\ell ; \mu}-\kappa^{\ell 2} \psi^{\ell *} \psi^{\ell}-G^{\ell}\left(\psi^{\ell *} \psi^{\ell}\right)\right\} \\
& +\frac{1}{8 \pi}\left[\left(\nabla \varphi+\frac{1}{\mathrm{c}} \partial_{t} \mathbf{A}\right)^{2}-(\nabla \times \mathbf{A})^{2}\right],
\end{aligned}
$$

where common summation convention is used for the index $\mu, G^{\ell}$ is a nonlinear selfinteraction function and

$$
\kappa^{\ell}=\frac{\omega^{\ell}}{\mathrm{c}}=\frac{m^{\ell} \mathrm{c}}{\chi}, \quad \omega^{\ell}=\frac{m^{\ell} \mathrm{c}^{2}}{\chi} .
$$

We assume that for every $\ell$ : (i) $m^{\ell}>0$ is the charge mass; (ii) $q^{\ell}$ is a real valued (positive or negative) charge; (iii) $\chi>0$ is a parameter similar to the Planck constant $\hbar$.

The gauges invariance of the system Lagrangian $\mathcal{L}$ yields for $\ell$-th charge a conserved 4-vector micro-current $J^{\ell \mu}=\left(\rho^{\ell} \mathrm{c}, \mathbf{J}^{\ell}\right)$ defined by the formulas (125) with natural substitutions $\psi \rightarrow \psi^{\ell}, m \rightarrow m^{\ell}$ and $q \rightarrow q^{\ell}$. The conservation/continuity equation takes the form

$$
\partial_{\nu} J^{\ell v}=0 \quad \text { or } \quad \partial_{t} \rho^{\ell}+\nabla \cdot \mathbf{J}^{\ell}=0, \quad \ell=1, \ldots, N .
$$

As a consequence of the continuity equations (155) the space integral of every $\rho^{\ell}(x)$ is a conserved quantity, which we assign to be exactly $q^{\ell}$, i.e. we assume the following charge normalization for $\ell=1, \ldots, N$ :

$$
\int_{\mathbb{R}^{3}} \frac{\rho^{\ell}(x)}{q^{\ell}} \mathrm{d} \mathbf{x}=-\frac{1}{m^{\ell} \mathrm{c}^{2}} \int_{\mathbb{R}^{3}}\left(\chi \operatorname{Im} \frac{\partial_{t} \psi^{\ell}}{\psi^{\ell}}+q^{\ell} \varphi\right)\left|\psi^{\ell}\right|^{2} \mathrm{~d} \mathbf{x}=1 .
$$

The Euler-Lagrange equations derived from $\mathcal{L}$ can be written in the form

$$
\begin{aligned}
& {\left[\mathrm{c}^{-2} \tilde{\partial}_{t}^{\ell 2}-\tilde{\nabla}^{\ell 2}+\kappa^{\ell 2}+G^{\ell \prime}\left(\left|\psi^{\ell}\right|^{2}\right)\right] \psi^{\ell}=0, \quad \ell=1, \ldots, N,} \\
& \nabla \cdot\left(\frac{1}{\mathrm{c}} \partial_{t} \mathbf{A}+\nabla \varphi\right)=-4 \pi \sum_{\ell} \rho^{\ell}, \\
& \nabla \times(\nabla \times \mathbf{A})+\frac{1}{\mathrm{c}} \partial_{t}\left(\frac{1}{\mathrm{c}} \partial_{t} \mathbf{A}+\nabla \varphi\right)=\frac{4 \pi}{\mathrm{c}} \sum_{\ell} \mathbf{J}^{\ell} .
\end{aligned}
$$

Equations (157)-(159) together with the normalization (156) constitute a complete set of equations describing the fields $\left\{\psi^{\ell}, \mathbf{E}, \mathbf{B}\right\}$. Observe that (158)-(159) are exactly the Maxwell equations written in terms of the field potentials, [23].

In any classical field theory over the four-dimensional continuum of space-time the energy-momentum tensor is of a fundamental importance. It provides the density of the energy, the momentum and the surface forces as well as the conservation laws that govern the energy and momentum transport. In this case we can naturally assign to every $\ell$-th particle its energy momentum tensor $T^{\ell \mu \nu}$ by formula (138) which together with energy momentum $\Theta^{\mu \nu}$ of the EM field satisfy the following conservations laws

$$
\partial_{\mu} T^{\ell \mu \nu}=f^{\ell v}, \quad \partial_{\mu} \Theta^{\mu \nu}=-f^{v},
$$


where $f^{\ell v}$ and $f^{v}$ are the Lorentz forces densities defined by

$$
f^{\ell \nu}=\frac{1}{\mathrm{c}} J_{\mu}^{\ell} F^{\nu \mu}, \quad f^{v}=\sum_{\ell} f^{\ell v}=\frac{1}{\mathrm{c}} J_{\mu} F^{v \mu}, \quad J_{\mu}=\sum_{\ell} J_{\mu}^{\ell} .
$$

It is worth to point out that it is the differential form of the energy-momentum conservation (160)-(161) which involve the densities of energy, momentum and forces rather than the original field equations are more directly related to corpuscular properties of the fields. In particular, for the charge model we study here the Lorentz force density arises in the differential form of the energy-momentum conservation equations and not in the original field equations. For detailed considerations of the structure and properties of the energymomentum tensor including its symmetry, gauge invariance and conservation laws we refer the reader to [6].

\subsection{Relations Between Relativistic and Non-relativistic Theories}

The time harmonic factor $\mathrm{e}^{-\mathrm{i} \omega_{0} t}$ which appears in $\omega_{0}$-static states as in (132) plays a very important role in this theory, including the nonrelativistic case. To reflect that we introduce a change of variables

$$
\psi(t, \mathbf{x}) \rightarrow \mathrm{e}^{-\mathrm{i} \omega_{0} t} \psi(t, \mathbf{x})
$$

and substitute it in the Lagrangian $L_{0}$ for a single charge defined by (153) with $N=1$ to obtain the Lagrangian $L_{\omega_{0}}$, which we call frequency shifted, it has the form

$$
\begin{aligned}
L_{\omega_{0}}\left(\psi, \psi^{*}, A^{\mu}\right)= & \frac{\chi}{2} \mathrm{i}\left(\psi^{*} \tilde{\partial}_{t} \psi-\psi \tilde{\partial}_{t}^{*} \psi^{*}\right) \\
& +\frac{\chi^{2}}{2 m}\left\{\frac{1}{\mathrm{c}^{2}} \tilde{\partial}_{t} \psi \tilde{\partial}_{t}^{*} \psi^{*}-\tilde{\nabla} \psi \tilde{\nabla}^{*} \psi^{*}-G\left(\psi^{*} \psi\right)\right\} \\
& +\frac{1}{8 \pi}\left[\left(\nabla \varphi+\frac{1}{\mathrm{c}} \partial_{t} \mathbf{A}\right)^{2}-(\nabla \times \mathbf{A})^{2}\right],
\end{aligned}
$$

where we use notation (152) with index $\ell$ omitted. The Lagrangian $L_{\omega_{0}}$ defined by the formula (163) is gauge and space-time translation invariant, it also invariant with respect to space rotations but it is not invariant with respect to the entire group of Lorentz transformations. Notice also that $\omega_{0}$-static states for the original Lagrangian defined by (153) turn into regular static states for the Lagrangian $L_{\omega_{0}}$, and that was one of the reasons to introduce it.

We describe now briefly how relativistic Lagrangian was modified to obtain nonrelativistic. The first step in this modification is the change of variables (162) which results in the construction of the frequency-shifted Lagrangian $L_{\omega_{0}}$ defined by (163). Then a gauge invariant and nonrelativistic Lagrangian $\hat{L}_{0}$ given by (7) is obtained from the Lagrangian $L_{\omega_{0}}$ by omitting in (163) the term $\frac{\chi^{2}}{2 m \mathrm{c}^{2}} \tilde{\partial}_{t} \psi \tilde{\partial}_{t}^{*} \psi^{*}$ and setting $\mathbf{A}=0$. To make transition from relativistic Lagrangian and equations to non-relativistic more transparent, we introduce here changes of variables allowing to recast the original field equations into a dimensionless form. These equations in dimensionless form allow to clarify three aspects of the theory for a single charge: (i) out of all the constants involved there is only one parameter of significance denoted by $\alpha$, and it coincides with the Sommerfeld fine structure constant $\alpha_{S} \simeq 1 / 137$ if $\chi=\hbar$ and $q, m$ are the electron charge and mass respectively; (ii) the non-relativistic Lagrangian (7) can be obtained from the relativistic one via the frequency-shifted Lagrangian 
(163) by setting there $\alpha=0$; (iii) the simultaneous fulfillment of charge and energy normalization conditions in relativistic case follows from smallness of $\alpha$.

Recall that the single charge nonrelativistic Lagrangian $\stackrel{\circ}{L}_{0}$ defined by (7) is constructed in Sect. 2.7 based on the relativistic one via the frequency shifted Lagrangian $L_{\omega_{0}}$ defined by (162)-(163). Let us introduce the following constants and new variables:

$$
\begin{aligned}
a_{\chi} & =\frac{\chi^{2}}{m q^{2}}, \quad \alpha=\frac{q^{2}}{\chi \mathrm{c}}, \quad \omega_{0}=\frac{m \mathrm{c}^{2}}{\chi}=\frac{\mathrm{c}}{\alpha a_{\chi}}, \\
\alpha^{2} \omega_{0} t & =\tau, \quad \mathbf{x}=a_{\chi} \mathbf{y}, \\
\psi(\mathbf{x}) & =\frac{1}{a_{\chi}^{3 / 2}} \Psi\left(\frac{\mathbf{x}}{a_{\chi}}\right), \\
\varphi(\mathbf{x}) & =\frac{q}{a_{\chi}} \Phi\left(\frac{\mathbf{x}}{a_{\chi}}\right), \quad \mathbf{A}(\mathbf{x})=\frac{q}{a_{\chi}} \mathrm{A}\left(\frac{\mathbf{x}}{a_{\chi}}\right) .
\end{aligned}
$$

In those new variables the field equations derived from the Lagrangian $L_{\omega_{0}}$ turn into the following dimensionless form:

$$
\begin{aligned}
& \alpha^{2}\left(\partial_{\tau}+\mathrm{i} \bar{\Phi}\right)^{2} \Psi-2 \mathrm{i}\left(\partial_{\tau}+\mathrm{i} \bar{\Phi}\right) \Psi-\left(\nabla_{y}-\mathrm{i} \alpha \overline{\mathrm{A}}\right)^{2} \Psi+\mathrm{G}^{\prime}\left(|\Psi|^{2}\right) \Psi=0, \\
& \frac{1}{4 \pi} \nabla_{y} \cdot\left(\alpha \partial_{\tau} \mathrm{A}+\nabla_{y} \Phi\right)=\left(\alpha^{2} \operatorname{Im} \frac{\partial_{\tau} \Psi}{\Psi}+\alpha^{2} \Phi\right)|\Psi|^{2}-|\Psi|^{2}, \\
& -\left(\nabla_{y} \times\left(\nabla_{y} \times \mathrm{A}\right)+\alpha \partial_{\tau}\left(\alpha \partial_{\tau} \mathrm{A}+\nabla_{y} \Phi\right)\right)=-4 \pi \alpha\left(\operatorname{Im} \frac{\nabla_{y} \Psi}{\Psi}+\alpha \overline{\mathrm{A}}\right)|\Psi|^{2} .
\end{aligned}
$$

Now we would like to show that the dimensionless form of the non-relativistic equations field equations (9), (10) can be obtained from the field equations (166) in the limit $\alpha \rightarrow 0$. To have a nonvanishing external magnetic field in the limit $\alpha \rightarrow 0$ we set

$$
\mathrm{A}_{\mathrm{ex}}=\alpha^{-1} \mathrm{~A}_{\mathrm{ex}}^{0} .
$$

Plugging in the expression (167) into (166) we obtain in the limit $\alpha \rightarrow 0$ the following dimensionless version of the field equations (9), (10):

$$
\begin{aligned}
\mathrm{i} \partial_{\tau} \Psi & =-\frac{1}{2}\left(\nabla_{y}-\mathrm{i} A_{\mathrm{ex}}^{0}\right)^{2} \Psi+\left(\Phi+\Phi_{\mathrm{ex}}\right) \Psi+\frac{1}{2} \mathrm{G}^{\prime}\left(|\Psi|^{2}\right) \Psi, \\
-\nabla_{y}^{2} \cdot \Phi & =4 \pi|\Psi|^{2}, \quad\left(\nabla_{y} \times\left(\nabla_{y} \times \mathrm{A}\right)\right)=0 .
\end{aligned}
$$

To get an insight in the nonrelativistic case as an approximation to the relativistic one we would like to make a few comments on the relative magnitude of terms that have to be omitted in (166) in order to obtain (168). The nonrelativistic case is defined as one when the charge velocity $v$ is much smaller than the speed of light $\mathrm{c}$, and a careful look at those omitted terms in (166) that have factors $\alpha$ and $\alpha^{2}$ shows that they can be small not only because of $\alpha$, but also because of the smallness of typical values of velocities compared to the speed of light. Indeed, every term that has factor $\alpha$ involves time derivatives with only one exception: the term $\alpha^{2}(\mathrm{i} \bar{\Phi})^{2} \Psi$. An estimation of the magnitude of the omitted terms for solutions of the form of wave-corpuscles (19) indicated that every such a term is of order $\alpha|\mathbf{v}| / \mathrm{c}$ where $\mathbf{v}$ is the wave-corpuscle velocity. The only omitted term in (166) which does not involve time derivatives is $\alpha^{2} \Phi^{2} \Psi$ and, in fact, it can be preserved in the nonrelativistic 
system which would be similar to (168) with properties analogous to (9). Analysis of that system is more involved and the treatment is similar to the one for the rest solution of the relativistic equation, [6].

\section{Relation to Quantum Theory}

While our neo-classical model is clearly different in setup from a conventional quantum model, the incorporation of nonlinear Schroedinger or Klein-Gordon equations on spacetime implies that some phenomena which are conventionally thought of as belonging to the realm of quantum theory properties, such as spectral lines of hydrogen or the interference pattern in the double slit experiment, which usually are computed with one-particle wave functions, may very well feature in our model as well. The following three subsections are meant as a first attempt to sort out which quantum phenomena may and which may not be accounted for by our model.

\subsection{Hydrogen Atom Model}

The purpose of this section is to introduce a hydrogen atom model within the framework of our non-relativistic theory for two interacting charges: an electron and a proton. We have no intend though to study this model in detail here but leave it as a subject for a separate paper. Our modest effort on the subject in this paper is, first, to indicate a similarity between our and Schrödinger's hydrogen atom models and to contrast it to any kind of Kepler model. Another point we can make based on our hydrogen atom model is that our theory does provide a basis for a regime of close interaction between two charges which differs significantly from the regime of remote interaction which is the primary focus of this paper and which was considered in detail in previous sections. Evidently, the results on interaction of many charges in the regime of remote interaction do not apply to the case of two charges in the hydrogen atom since they are in close proximity and the potentials can vary significantly over their locations. Hence, other methods have to be developed for the hydrogen atom model.

To model interaction of two charges at a short distance we must consider the original system (77) for two charges with $-q_{1}=q_{2}=q>0$, that is

$$
\begin{aligned}
\mathrm{i} \chi \partial_{t} \psi_{1} & =-\frac{\chi^{2} \nabla^{2} \psi_{1}}{2 m_{1}}-q\left(\varphi_{1}+\varphi_{2}\right) \psi_{1}+\frac{\chi^{2} G_{1}^{\prime}\left(\left|\psi_{1}\right|^{2}\right) \psi_{1}}{2 m_{1}}, \\
-\nabla^{2} \varphi_{1} & =-4 \pi q\left|\psi_{1}\right|^{2} \\
\mathrm{i} \chi \partial_{t} \psi_{2} & =-\frac{\chi^{2} \nabla^{2} \psi_{2}}{2 m_{2}}+q\left(\varphi_{1}+\varphi_{2}\right) \psi_{2}+\frac{\chi^{2} G_{2}^{\prime}\left(\left|\psi_{2}\right|^{2}\right) \psi_{2}}{2 m_{2}}, \\
\nabla^{2} \varphi_{2} & =-4 \pi q\left|\psi_{2}\right|^{2}
\end{aligned}
$$

Note that the model describes proton-electron interaction if $q=e$ is the electron charge, $\chi=\hbar$ is the Planck constant, $m_{1}$ and $m_{2}$ are the electron and the proton masses respectively. Let us look now at time-harmonic solutions to the system (169) in the form

$$
\begin{aligned}
\psi_{1}(t, \mathbf{x}) & =\mathrm{e}^{-\mathrm{i} \omega_{1} t} u_{1}(\mathbf{x}), \quad \psi_{2}=\mathrm{e}^{-\mathrm{i} \omega_{2} t} u_{2}(\mathbf{x}), \\
\Phi_{1} & =-\frac{\varphi_{1}}{q}, \quad \Phi_{2}=\frac{\varphi_{2}}{q} .
\end{aligned}
$$


The system (169) for such solutions turns into the following nonlinear eigenvalue problem:

$$
\begin{aligned}
& -\frac{a_{1}}{2} \nabla^{2} u_{1}+\left(\Phi_{1}-\Phi_{2}\right) u_{1}+\frac{a_{1}}{2} G_{1}^{\prime}\left(\left|u_{1}\right|^{2}\right) u_{1}=\frac{\chi}{q^{2}} \omega_{1} u_{1}, \\
& -\frac{a_{2}}{2} \nabla^{2} u_{2}+\left(\Phi_{2}-\Phi_{1}\right) u_{2}+\frac{a_{2}}{2} G_{2}^{\prime}\left(\left|u_{2}\right|^{2}\right) u_{2}=\frac{\chi}{q^{2}} \omega_{2} u_{2}, \\
& \text { where } a_{1}=\frac{\chi^{2}}{q^{2} m_{1}}, a_{2}=\frac{\chi^{2}}{q^{2} m_{2}} .
\end{aligned}
$$

Here $a_{1}$ coincides with Bohr radius. We seek solutions of (171) satisfying the charge normalization conditions

$$
\int_{\mathbb{R}^{3}}\left|u_{1}\right|^{2} \mathrm{~d} \mathbf{x}=1, \quad \int_{\mathbb{R}^{3}}\left|u_{2}\right|^{2} \mathrm{~d} \mathbf{x}=1
$$

The potentials $\Phi_{i}$ are presented using (169) as follows:

$$
\Phi_{i}=4 \pi\left(-\nabla^{2}\right)^{-1}\left|u_{i}\right|^{2}=\int_{\mathbb{R}^{3}} \frac{\left|u_{i}\right|^{2}(\mathbf{y})}{|\mathbf{y}-\mathbf{x}|} \mathrm{d} \mathbf{y}, \quad i=1,2 .
$$

Let us introduce now the following energy functional

$$
\begin{aligned}
\mathcal{E}\left(u_{1}, u_{2}\right)= & q^{2} \int_{\mathbb{R}^{3}}\left\{\frac{a_{1}\left[\left|\nabla u_{1}\right|^{2}+G_{1}\left(\left|u_{1}\right|^{2}\right)\right]}{2}+\frac{a_{2}\left[\left|\nabla u_{2}\right|^{2}+a_{2} G_{2}\left(\left|u_{2}\right|^{2}\right)\right]}{2}\right. \\
& \left.-\left(\Phi_{1}-\Phi_{2}\right)\left(\left|u_{2}\right|^{2}-\left|u_{1}\right|^{2}\right)-\frac{\left|\nabla\left(\Phi_{1}-\Phi_{2}\right)\right|^{2}}{8 \pi}\right\} \mathrm{d} \mathbf{x},
\end{aligned}
$$

where $\Phi_{1}, \Phi_{2}$ are determined in terms of $u_{1}, u_{2}$ by (173). Notice that (171) describe stationary points of the energy functional $E$ and can be obtained by its variation under constraints (172) with the frequencies $\omega_{1}, \omega_{2}$ being the Lagrange multipliers. Hence variational methods can be applied to study the nonlinear eigenvalue problem (171)-(172). Our analysis is based on two points. First, the hydrogen linear Schrödinger operator's negative eigenvalues, which describe hydrogen spectrum, coincide with the critical points of corresponding quadratic functional $\mathcal{E}_{\text {lin }}\left(u_{1}\right)$ under the first constraint of (172), the eigenvalues can be determined by a min-max method. The second point is that under certain conditions, when applying the min-max method to the nonlinear functional $\mathcal{E}\left(u_{1}, u_{2}\right)$ subjected to the constraints, we can approximate it by $\mathcal{E}_{\text {lin }}\left(u_{1}\right)+C$ with a certain constant $C$. The smallness of the ratio $m_{1} / m_{2} \cong 1 / 1836$ of electron to proton masses plays an important role in the analysis, this smallness implies that for the critical points with low energies the potential $\Phi_{2}$ of the proton is close to the Coulomb's potential $1 /|\mathbf{x}|$ at spatial scales of the order $a_{1}$. If one assumes the characteristic size $a_{1}$ of a free electron is 10-100 times larger then the Bohr radius, then our preliminary analysis shows that the energy levels of our model for hydrogen atom are in a good agreement with the classical spectral theory of hydrogen. As to the assumption that a free electron significantly contracts in size when it is bound to a proton, it seems to us to be quite reasonable. Note also that the existence of the discrete energy levels of nonlinear functionals under certain general restrictions is well known. Since the energy functional and the constraints are invariant with respect to multiplication by -1 , one can apply the Lusternik-Schnirelman theory which guarantees the existence of an infinite set of critical points under appropriate conditions see, for example, [21, 28] and references therein. 
Note that (171) provide a preliminary model for hydrogen atom and we need to modify the way electrostatic compensation is introduced. The reason for the modification can be seen from the first equation (171) for the electron. Our analysis shows that since $m_{1} / m_{2}$ is small, when we consider levels of the energy we can replace with a good accuracy controlled by $m_{1} / m_{2}$ the energy $\mathcal{E}\left(u_{1}, u_{2}\right)$ of the proton-electron system by energy of the electron with the potential of the proton electrostatic field replaced by Coulomb potential $\frac{1}{|\mathbf{x}|}$ :

$$
\mathcal{E}_{1}\left(u_{1}\right)=q^{2} \int_{\mathbb{R}^{3}} \frac{a_{1}\left[\left|\nabla u_{1}\right|^{2}+G_{1}\left(\left|u_{1}\right|^{2}\right)\right]}{2}+\left(\Phi_{1}-\frac{1}{|\mathbf{x}|}\right)\left|u_{1}\right|^{2}-\frac{\left|\nabla \Phi_{1}\right|^{2}}{8 \pi} \mathrm{d} \mathbf{x} .
$$

The equation for the electron takes then the form

$$
\begin{aligned}
& -\frac{a_{1}}{2} \nabla^{2} u_{1}+\left(\Phi_{1}-\frac{1}{|\mathbf{x}|}\right) u_{1}+\frac{a_{1}}{2} G_{1}^{\prime}\left(\left|u_{1}\right|^{2}\right) u_{1}=\frac{\chi}{q^{2}} \omega_{1} u_{1}, \\
& \Phi_{1}=\int_{\mathbb{R}^{3}} \frac{\left|u_{1}\right|^{2}(\mathbf{y})}{|\mathbf{y}-\mathbf{x}|} \mathrm{d} \mathbf{y} .
\end{aligned}
$$

The energy $\mathcal{E}_{1}\left(u_{1}\right)$ looks like the energy of $\mathcal{E}_{\text {lin }}\left(u_{1}\right)$ of the linear Schrodinger hydrogen model, with two differences: nonlinear term $G_{1}\left(\left|u_{1}\right|^{2}\right)$ and electrostatic self-interaction term $\Phi_{1}\left|u_{1}\right|^{2}$. Note that for a free electron these two terms exactly compensate one another producing the equilibrium. We can show that if the size parameter $a$ in $G_{1}^{\prime}=G_{1, a}^{\prime}$ is large compared with the Bohr radius $a_{1}$, the contribution of nonlinear term to energy levels is small. At the same time, the electrostatic self-interaction term $\Phi_{1}\left|u_{1}\right|^{2}$ gives a contribution which preserves discreteness of the lower energy levels, but we cannot make it arbitrary small by variation of the size parameter $a$. To be able to obtain quantitative agreement with the Schrödinger hydrogen model energy levels, we need to modify electrostatic compensation. We need to define it in such a way that it completely compensates electrostatic repelling not only in the regime of distant interaction but in all regimes.

\subsection{Brief Comparison with the Schrödinger Wave Mechanics}

The nonrelativistic version of our wave mechanics has many features in common with the Schrödinger wave mechanics. In particular, the charges wave functions are complex valued, they satisfy equations resembling the Schrödinger equation, the charge normalization condition is the same as in the Schrödinger wave mechanics. Our theory provides for a hydrogen atom model which has a lot in common with that of Schrödinger, but its detailed study is outside of the scope of this article. There are features of our wave theory though that distinguish it significantly from the Schrödinger wave mechanics, and they are listed below with more detailed considerations available in [6].

- The single-particle wave function is interpreted as a material wave, as in Schrödinger's original thinking, and not as a probability amplitude in the sense of Born.

- Every single-particle Lagrangian has a nonlinear self-interaction term providing for a cohesive force holding it together as it moves freely or accelerates.

- A single charge either free or in external EM field is described by a soliton-like wave function parametrized by the position and the momentum related to the corresponding point mechanics. It propagates in the space without dispersion even when it accelerates, and this addresses one of the above mentioned "grave difficulties" with the Schrödinger's interpretation of the wave function expressed by M. Born. 
- Charges are always coupled with and inseparable from the EM field.

- When dressed charges are separated by distances considerably larger than their sizes, their wave functions and the corresponding EM fields maintain soliton-like representation.

- The correspondence between the wave mechanics and a point mechanics comes through the closed form soliton-like representation of wave functions in which point mechanics positions and momenta enter as parameters. In particular, the wave function representation includes the de Broglie wave vector as an exact parameter, it equals (up to the Planck constant) the point mechanics momentum. In addition to that, the corresponding group velocity matches exactly the velocity of a soliton-like solution and the point mechanics velocity.

- The orthodox Schrödinger wave equation is linear, and any freely propagating wavepacket spreads out with time, whereas our wave corpuscle preserves its shape. Though the spatial spread of a wave corpuscle is non-zero, the velocity of wave corpuscle is precisely defined, indicating that Heisenberg uncertainty principle between the position and momentum of a particle cannot be a universal principle in our model.

- In the case of many interacting charges every charge is described by its own wave function over the same three dimensional space in contrast to the Schrödinger wave mechanics for many charges requiring multidimensional configuration space.

- Our theory has a relativistic version based on a local, gauge and Lorentz invariant Lagrangian with most of the above listed features.

\subsection{Quantum Statistics and Non-locality}

In this subsection, we address the question whether the quantum statistical predictions could possibly arise from our model.

A key element of the WCM that has not been studied yet is the regime of a time limited close interaction. More precisely, it is the regime when initially free moving charge undergoes for a naturally limited time a close interaction with another charge or an external EM field after which the charge continues to move freely again but with altered location and velocity. A typical example of such an interaction is when one moving charge is scattered by another one, or when a charge passes through a bounded domain in the space with a strong external EM field. Let us try to imagine what can happen according to the WCM to a charge during a time limited close interaction. We recall the reader that in the WCM when charges are far apart every charge is represented by a particle-like well localized wave function as in (19) as a result of a fine balance of forces including the nonlinear self-interaction. Importantly, the cohesive action of the nonlinear self-interaction is very subtle, and by no means it is a brute attractive force since there is no action at distance in the WCM. Now, when one charge comes close to another or if it enters a domain with an external EM field varying at a sufficiently small scale, a fine balance of forces holding the charge together is disrupted. We can already see consequences of such a disruption in the WCM hydrogen atom model in Sect. 4.1 where possibly the electron size reduces by a factor of order 10-100 compared to that of the free electron under attractive action of a single proton. A disruption of the subtle cohesive action of the nonlinear self-interaction can also cause the charge wave function to spread out substantially, obtain a smaller amplitude and become wave-like. We can imagine further that during the time of interaction the evolution of the extended wave function is determined by an interplay of two factors: (i) the linear Schrödinger component of the field equation; (ii) its nonlinear component due the nonlinear self-interaction. Shortly after the interaction ends the wave-function of the charge restores the particle-like form but its position and velocity after the contraction will depend sensitively on details of the interaction. 
So, effectively, a limited time interaction switches one particle-like state of the charge to another.

Based on the above hypothetical features of a time limited close interaction one can explain how the entirely deterministic WCM can conceivably lead to some of probabilistic aspects of the QM. Suppose for the sake of argument that the time scale of details of the interaction process is smaller than an observer can resolve, and, consequently, he sees the interaction result as a transition from one particle-like state to another. The interaction process can alter the total momentum of the charge quite considerably. This momentum alteration combined with effects of the nonlinear self-interaction can cause an extreme sensitivity to the initial data and that in turn can make the transition look like it is random and, hence, a subject to a statistical theory. An interesting feature of the nonlinear self-interaction in the WCM that might be relevant to the extreme sensitivity is that it is not analytic and singular for small wave-amplitudes (see examples of the WCM nonlinearity in Sect. 2.4). Consequently, small wave amplitudes can play far more important role in the WCM than in the case of conventional nonlinearities which are analytic for small amplitudes. Going further we observe that the WCM field equations are similar to the Schrödinger equation. Hence, it is conceivable that the statistics of the transition will be determined with certain degree of accuracy by a wave function satisfying an effective linear Schrödinger equation. Some general ideas on the "determinism beneath quantum mechanics" at the Planck scale were put forward recently by 't Hooft (see [43] and references therein).

Let us use the described above hypothetical scenario of interaction to explain the doubleslit experiment. Suppose that a single electron is fired by a device and moves freely as a particle-like wave toward a double-slit apparatus. As the electron approaches and interacts with the double-slit apparatus its wave function spreads out quite substantially, its amplitude reduces and consequently the electron turns into a "real wave". This extended wave penetrates through the both slits and over a limited time leaves the apparatus. Being outside of the apparatus in a free space the electron wave function contracts back to its particle-like shape and proceeds toward a sensitive screen until it hits it at a well defined impact location. Assume for the sake of argument that the initial condition of the fired electron can not be controlled with a sufficient accuracy (which may be higher than in the existing experiments). Then, the impact location can appear to be random with a statistics consistent with well known interference pattern as described in the modern double-slit experiments, [19, Sect. 1.1].

Another qualitatively important regime is the regime of close interaction for an extended or even infinite period of time. This regime can occur, in particular, in complex systems involving many charges such as atoms, molecules or solids. As we have already indicated in Sect. 4.1 the WCM hydrogen atom has features which are very similar the Schrödinger one. In particular, the primary binding force in that model between the electron and the nucleus is the EM attraction. As to solids, let us briefly recall basics of their treatment in the QM. As any theory of many particles, the fundamental QM theory of a solid is of enormous complexity, but the standard simplified QM treatment of charges in crystalline solids is based on a free-electron model with the following basics assumptions, [2, Chap. 1]: (i) positively charged ionized atoms, consisting of nuclei and tightly bound to them "core electrons", form an immobile periodic lattice structure; (ii) "valence electrons" called also conductance electrons are "allowed to wander far away from their parent atoms"; (iii) the conductance electrons are non-interacting and independent and the interaction between a conductance electron and the periodic lattice is modeled via a periodic potential. Such a simplified QM theory is effectively reduced to the one-electron theory for the Schrödinger operator with the periodic potential. Consequently, the eigenfunctions of such an operator are of the Bloch 
form and are extended over the entire crystal. The fundamental WCM theory of a solid is of an enormous complexity as well, but similarly to the QM theory we can introduce a simplified WCM model for a solid based on the same assumptions as in the QM theory. Hence, as in the QM model there is an immobile periodic lattice of ionized atoms described by a periodic potential corresponding to an external electric field. The one-electron WCM model is similar to the QM one, but it differs from it by the presence of nonlinear selfinteraction. In this non-relativistic WCM model a mobile conductance/valence electron is subjected to (i) attractive forces of the periodic lattice; (ii) electric force of its own electric field; (iii) nonlinear-self interaction forces. Since in a solid the distance between atoms is pretty small, it is of order the atom Bohr radius, the cohesive action of the nonlinear selfinteraction can be disrupted and the wave function can spread out significantly and even it might resemble a Bloch eigenmode, in which case the electron would occupy the entire crystal sample.

The above considerations bring us naturally to issues of the charge size and the WCM theory locality. As the above considerations suggest in the WCM the electron size can vary significantly depending on whether it is free or if it is bound in a atom, or if it is a conductance electron in a crystalline solid. In particular, the size of the electron can increase dramatically when it undergoes a strong close interaction with an external EM field or a system of other charges. We expect a free electron to have size more than 20 times larger than Bohr radius, which would lead to an interaction with many hundreds of atoms when it approaches a crystal lattice even in the beginning of interaction when it still preserves its size. As to the locality of the WCM it is perfectly local in one sense but can be non-local in another sense. Namely, the WCM theory is perfectly local in the sense that there is no action of distance. But the charge evidently is not perfectly local since in the WCM it is not a point but at the best a localized wave which can spread out significantly. Consequently, it is conceivable within the WCM that an elementary charge being a spatially distributed quantity can be simultaneously at two distant spatial locations, and in this sense the WCM might deviate significantly from being a local theory.

One can also wonder what is a relation between the WCM and hidden variables theories, see [18, Sect. 12.2], [22, Sects. 1.5, 3.7.2], and a review article [16] with references therein. Particularly, it is interesting to look how does the WCM compare with the Bohmian Mechanics (BM), [10], [22, Sects. 3.1, 3.2], [15], [14, Sect. 8], a well known example of hidden variables theories. Even a brief look on the WCM and the BM shows their significant differences: (i) in the BM an elementary charge is a point whereas in the WCM it is a distributed quantity, a wave; (ii) the WCM theory is local in the sense that there is no action at distance, and it is no so for the BM; (iii) the WCM is a genuine Lagrangian Mechanics and, consequently, the Third Newton Law is always satisfied, and it is not so for the BM, [22, Sect. 3.3.2]. In addition to that, as we have already indicated above, the WCM might account for the QM statistics via the dynamic instability approximately, and the verification of that, including the accuracy of approximation, is a subject of future studies. But it is absolutely clear already that the statistical predictions of the WCM can not be precisely the same as those of the QM, since the WCM field equations might only approximately and under certain conditions produce the QM evolution equation. The later factor evidently differs the WCM from the BM in which the Schrödinger equation is an exact equation for the wave function as a part of the BM variables.

Acknowledgements The research was supported through Dr. A. Nachman of the U.S. Air Force Office of Scientific Research (AFOSR), under grant number FA9550-04-1-0359. The authors are very grateful to Michael Kiessling for reading the manuscript and suggestions which helped to improve the exposition. 
Open Access This article is distributed under the terms of the Creative Commons Attribution Noncommercial License which permits any noncommercial use, distribution, and reproduction in any medium, provided the original author(s) and source are credited.

\section{References}

1. Appel, W., Kiessling, M.: Mass and spin renormalization in Lorentz electrodynamics. Ann. Phys. 289, 24-83 (2001)

2. Ashcroft, N., Mermin, N.: Solid-State Physics. Harcourt College Publisher, San Diego (1976)

3. Babin, A., Figotin, A.: Linear superposition in nonlinear wave dynamics. Rev. Math. Phys. 18(9), 9711053 (2006)

4. Babin, A., Figotin, A.: Wavepacket preservation under nonlinear evolution. Commun. Math. Phys. 278, 329-384 (2008)

5. Babin, A., Figotin, A.: Nonlinear dynamics of a system of particle-like wavepackets. In: Bardos, C., Fursikov, A. (eds.) Instability in Models Connected with Fluid Flows. International Mathematical Series, vol. 6. Springer, Berlin (2008)

6. Babin, A., Figotin, A.: Wave-corpuscle mechanics for elementary charges. e-print available online at arXiv:0812.2686

7. Barut, A.: Electrodynamics of and Classical Theory of Fields and Particles. Dover, New York (1980)

8. Benci, V., Fortunato, D.: Solitary waves in the nonlinear wave equation and in gauge theories. J. Fixed Point Theory Appl. 1, 61-86 (2007)

9. Bialynicki-Birula, I., Mycielski, J.: Nonlinear wave mechanics. Ann. Phys. 100, 62-93 (1976)

10. Bohm, D., Hiley, B.: The Undivided Universe: An Ontological Interpretation of Quantum Theory. Routledge, London (1993)

11. Born, M.: Atomic Physics, 2nd edn. Blackie, Glasgow (1937)

12. Born, M., Infeld, L.: Foundations of the new field theory. Proc. R. Soc. Lond. Ser. A 144(852), 425-451 (1934)

13. De Broglie, L.: Heisenberg's Uncertainties and the Probabilistic Interpretation of Wave Mechanics. Kluwer, Dordrecht (1990)

14. Dürr, D., Teufel, S.: Bohmian Mechanics: The Physics and Mathematics of Quantum Theory. Springer, Berlin (2009)

15. Dürr, D., Goldstein, S., Zanghì, N.: Quantum equilibrium and the origin of absolute uncertainty. J. Stat. Phys. 67, 843-907 (1992)

16. Genovese, M.: Research on hidden variable theories: A review of recent progresses. Phys. Rep. 413, 319-396 (2005)

17. Goldstein, H., Poole, C., Safko, J.: Classical Mechanics, 3rd edn. Addison-Wesley, Reading (2000)

18. Gottfried, K., Yan, T.: Quantum Mechanics: Fundamentals, 2nd edn. Springer, Berlin (2003)

19. Greenstein, G., Zajonc, A.: The Quantum Challenge, 2nd edn. Jones and Bartlett, Boston (2005)

20. Griffiths, D.: Introduction to Elementary Particles. Wiley, New York (1987)

21. Heid, M., Heinz, H., Weth, T.: Nonlinear eigenvalue problems of Schrödinger type admitting eigenfunctions with given spectral characteristics. Math. Nachr. 242, 91-118 (2002)

22. Holland, P.: The Quantum Theory of Motion. Cambridge University Press, Cambridge (1995)

23. Jackson, J.: Classical Electrodynamics, 3rd edn. Wiley, New York (1999)

24. Kato, T.: Nonlinear Schrödinger equations. In: Holden, H., Jensen, A. (eds.) Schrödinger Operators. Lecture Notes in Physics, vol. 345. Springer, Berlin (1989)

25. Kiessling, M.: Electromagnetic field theory without divergence problems 1. The born legacy. J. Stat. Phys. 116, 1057-1122 (2004)

26. Kiessling, M.: Quantum Abraham models with de Broglie-Bohm laws of quantum motion. e-print available online at arXiv:physics/0604069v2

27. Komech, A.: Lectures on Quantum Mechanics (nonlinear PDE point of view). Lecture Notes of the Max Planck Institute for Mathematics in the Sciences, LN 25/2005, Leipzig (2005). http://www.mis.mpg.de/ preprints/ln/lecturenote-2505-abstr.html

28. Lions, P.-L.: Solutions of Hartree-Fock equations for Coulomb systems. Commun. Math. Phys. 109(1), 33-97 (1987)

29. Møller, C.: The Theory of Relativity, 2nd edn. Oxford (1982)

30. Morse, P., Feshbach, H.: Methods of Theoretical Physics, vol. I. McGraw-Hill, New York (1953)

31. Nodvik, J.S.: A covariant formulation of classical electrodynamics for charges of finite extension. Ann. Phys. 28, 225 (1964)

32. Pauli, W.: General Principles of Quantum Mechanics. Springer, Berlin (1980) 
33. Pauli, W.: Theory of Relativity. Dover, New York (1981)

34. Pearle, P.: Classical electron models. In: Teplitz, D. (ed.) Electromagnetism Paths to Research, pp. 211295. Plenum, New York (1982)

35. Poincaré, H.: Comptes Rend. 140, 1504 (1905)

36. Poincaré, H.: Rend. Circ. Mat. Palermo 21, 129-176 (1906)

37. Rohrlich, F.: Classical Charged Particles, 3rd edn. Addison-Wesley, Reading (2007)

38. Schiff, L.: Quantum Mechanics. McGraw-Hill, New York (1949)

39. Schrödinger, E.: Collected Papers on Wave Mechanics and Four Lectures on Wave Mechanics. Chelsea, New York (1982)

40. Schwinger, J.: Electromagnetic mass revisited. Found. Phys. 13(3), 373-383 (1983)

41. Spohn, H.: Dynamics of Charged Particles and Thier Radiation Field. Cambridge Univ. Press, Cambridge (2004)

42. Sulem, C., Sulem, P.: The Nonlinear Schrödinger Equation. Self-focusing and Wave Collapse. Springer, Berlin (1999)

43. 't Hooft, G.: Determinism beneath quantum mechanics. In: Elitzur, A., et al. (eds.) Quo Vadis Quantum Mechanics. Springer, Berlin (2005)

44. von Laue, M.: Phys. Z. 12, 85 (1911)

45. Weinberg, S.: Testing quantum mechanics. Ann. Phys. 194, 336-386 (1989)

46. Wentzel, G.: Quantum Theory of Fields. Dover, New York (2003)

47. Weyl, H.: Space-Time-Matter. Dover, New York (1922)

48. Yaghjian, A.: Relativistic Dynamics of a Charged Sphere: Updating the Lorentz-Abraham Model, 2nd edn. Springer, Berlin (2006)

49. Yaghjian, A.: Absence of a consistent classical equation of motion for a mass-renormalized point charge. Phys. Rev. E 78, 046606 (2008) 